\title{
DISTRIBUCIÓN ESPACIAL DE LOS ADEPHAGA ACUÁTICOS (COLEOPTERA) EN LA CUENCA DEL RÍO SEGURA (SE DE LA PENÍNSULA IBÉRICA).
}

\author{
A. Millán, J. Velasco, M.L Suárez, M.R. Vidal-Abarca y L. Ramírez-Díaz. \\ Departamento de Ecología c Hidrología, Facultad de Biología, Universidad Murcia. Cainpus Universitario de Espinardo. 30100. \\ Murcia (Spain).
}

Palabras clave: Coleópteros Adephaga acuáticos, técnicas de ordenación, cuenca del Segura

Key words: Aquatic Adephaga, ordination methods, Segura basin.

\begin{abstract}
SPATIAL DISTRIBUTION OF AQUATIC ADEPHAGA (COLEOPTERA) IN SEGURA RIVER BASIN (SE IBERIAN PENINSULA).

Thrce ordination techniques. Multiple Correspondeiicc Analysis (MCA), Multiple Canonical Correspondence Analysis (MCCA) and Correspondence Analysis (CA), were applied to characterizc the spatial distribution of the 64 species of aquatic Adephaga (Coleoptera) found in the Segura River basin. All threc ordination inethods gave similar results, being altitude, mineralization of the water, and riparian vegetation the rnost important environmental factors determining the composition of the asscmblages. A clear gradient from the head waters to the river mouth was detccted, defined within a very heterogeneous spatial framework.

The analysis of a contingence table with CA, used as a coinplementary aid to the interpretation of the MCCA, allowed a better understanding of the relationship betwcen the species and the environrnentnl variables. Four spccics groups were defined according to the results: 1. typical head-water species; 2 , species in the inedium plane oí'the river basin; 3 , less mineralized water bodies in the low reach of the river; and 4 highly inineralized water bodies in the low reach of the river. Some pioneer and opportunistic species, showing a highly spatial plasticity, were found among all groups, reducing their differences and contributing to the establishment of a continuous biological gradicnt closcly rclated with the environrnental gradiente.
\end{abstract}

\section{INTRODUCCIÓN}

Distintos trabajos han deinostrado que el estudio por separado, de las comunidades de Coleópteros acuáticos, refleja las mismas condiciones ambientales que la comunidad de macroinvertebrados en su conjunto (p.ej. JEFFRIES, 1988), pero con ventajas adicionales como: el gran espectro de hábitats que son capaces de caracterizar, su capacidad para ocupar todos o casi todos los rangos de los parámetros ambientales seleccionados, su facilidad de muestreo y la amplia bibliografía disponible. Adcmás, ciertas especies de Adcphaga acuáticos, pueden indicar grados de contaminación y contenido de oxígeno disuelto (HEUSS, 1989).
Otro aspecto iinportante, al que no se le ha prestado mucha atención en la literatura. es la larga vida de los adultos, que permite encontrarlos durante gran parte del año, disminuyendo así la variabilidad estacional en las muestras (EYRE et al.. 1986. EYRE \& FOSTER, 1989), y por tanto el esfuerzo de inuestreo.

Por ello. no resulta averiturado catalogar a los colcópteros como buenos indicadoses de las características ainbicntales de los hábitats acuáticos que ocupan (BRANCUCCI. 1980) y. sobre todo, como importantes descriptores de los cambios espaciales y temporales producidos en los sistemas acuáticos (RICHOUX \& CASTELLA, 1986; RICHOUX. 1988).

La investigación en ecología de este grupo de insectos está confinada. normalmente, a tipos similares de hábitats o a áreas 
muy restringidas (p. cj. EYRE et al. 1986), con estudios que relacionan las características del medio con la presencia de determinadas especies de macroinvcrtebrados acuáticos. Estos estudios se pueden diferenciar, fundamentalmente, en dos tipos:

a) Estudios de carácter descriptivo con un tratamiento de los datos sencillo. donde se asocian, a través de documentación hibliográfica y de la experiencia de campo. las variables abióticas y/o bióticas del medio con las cspecics encontradas (HOSSEINIE. 1078; HIESIADKA. 1980: BAGGE. 1983: MIELEWCZYK, 1983: HOURASSA et al., 1986; BUSSLER. 1988: HANSON \& SWANSON, 1989).

b) Estudios donde se aplican técnicas estadísticas y/o multivariantes (LARSON, 1985; LANCASTER \& SCUDDER, 1987; FRIDAY, 1987; EYRE \& RUSHTON, 1989: FOSTER et al. 1990) cuya finalidad es interpretar de forma objetiva las tendencias de distribución y asociación de cspecics cori las estaciones de mucstreo y variables del medio.

En la Península Ibérica sólo los trabajos de SOLER (1972), MONTES et al. (1980) y. recientemente, los de RIBERA \& FOSTER (1992). RIBERA et al. (1995 a y b) y MORENO $e t$ al.. (1997) cmplcan técnicas estadísticas más complejas. El presente estudio, siguiendo esta línea, utiliza diferentes técnicas de ordenación con la finalidad de determinar la que define mejor los patrones de distribución general y las variables abióticas y bióticas asociadas a las especies de Adephaga acuáticos en la cuenca del río Segura.

\section{ÁREA DE ESTUDIO}

La cuenca del río Segura, con $14.432 \mathrm{Km}^{2}$, es la tercera inás importante de la vertiente mediterránea. Sc extiende sobre áreas geográficas de las provincias de Granada, Jaén, Almería. Albacete, Murcia y Alicante (Fig. 1).

Las Cordilleras Béticas limitan esta cuenca por el norte y oeste. separándola por el ocstc de la cuenca del Guadalquivir. Al suroeste queda limitada por la Cordillera Prelitoral Murciana: por el este. la sierra del Carche la separa de la cuenca clcl Vinalopó. y por el norte, la sierra de la Pinilla y parte de la sierra de Alcaraz, la separan de la cuenca del Júcar.

El cje principal de la cuenca lo constituye el río Segura, que nace cii la sierra del mismo nornbrc eri la provincia de Jaén. a $1.412 \mathrm{~m}$ de altitud. Tras recorrer $325 \mathrm{Km}$ desemboca en el Mediterráneo, en Guardamar del Segura (Alicante).

Los principales afluentes del Segura, con aportes continuos de agua, son los ríos Madera, Tus y Mundo por la margen izquierda y los ríos Zumeta y Taibilla por la derecha. Otros afluentes de menor importancia, al presentar nportcs de caudales intermitentes. son las ramblas de Agua Amarga, Ju- dío, Moro, Tinajón, Santomera y el río Chícamo por la inargen izquierda; y los ríos Moratalla, Argos, Quípar, Mula y Guadalentín por la margen derecha.

Ambientalmente, la cuenca del río Segura sc caracteriza por su aridez (VIDAL-ABARCA et al. 1992). con largos períodos de déficit hídrico, pero con precipitaciones puntuales de carácter torrencial, y una geología y litología complejos. Presenta uri gran núincro de arroyos temporales o fluctuantes. en general muy mineralizados y cutrofizados. Otro aspecto importante a destacar es la enorme demanda de agua para riego y la elevada contaminación orgánica de un gran número de ambientes, que somete a la comunidad de macroinvertebrados acuáticos. sobre todo a partir de la vega media y baja, a situaciones de fuerte estrés ambiental. Todo ello confiere a la Lona de cstudio unas enracterísticas peculiares y de gran interés desdc el punto de vista ecológico.

\section{METODOLOGÍA}

\section{Toma de muestras}

El estudio se ha basado en la prospección de 390 estaciones de muestreo durante iin período de aproximadamente 11 años (1980-90), con el fin de recoger la mayor riqueza de cspecics. Los muestreos se realizaron estacionalmente en la rnayoría de los casos (MILLAN el al., 1992). Para la recogida de organismos se utilizó una manga entomológica de $30 \mathrm{~cm}$ de lado y 1 $\mathrm{mm}$ de luz de malla. hasta que no se observaban nuevas especies. Los muestreos se completaron con el uso de un colador pequeño para zonas intersticiales o de dimensiones reducidas.

Simultaneamente, se tomaron datos de 13 variables del incdio. seis físicas (altitud, persistencia del agua, profundidad, velocidad dc la coincntc, sustrato dominante y tipo de sedimento); tres biológicas (macrólitos dominantes, cobertura de macrófitos y vegetación de ribera); y cuatro químicas (grado de mineralización-conductividad, tipo de mineralización, oxígeno disuelto y contaminación orgánica). En VIDAL-ABARCA (1985) aparece una relación detallada de las tćcnicas empleadas para la medida de dichos parámetros.

\section{Análisis de los datos}

Con las 64 especies de Hydradefaga encontradas (Apéndice 1) en un total de 248 estaciones (MILLAN, 1991). se claboró una matriz de presencia/ausencia de 248 estaciones x 64 especies. Se ha preferido utilizar datos de presencia/ausencia, dado el carácter extensivo del estudio, la marcada heterogencidad de las muestras y las diferentes características de los ciclos de vida de las especies estudiadas. A esta primera matriz se le aplicó un Análisis de Correspondencias Múltiples (MCA). Esta lécnica es una generalización del Análisis de 


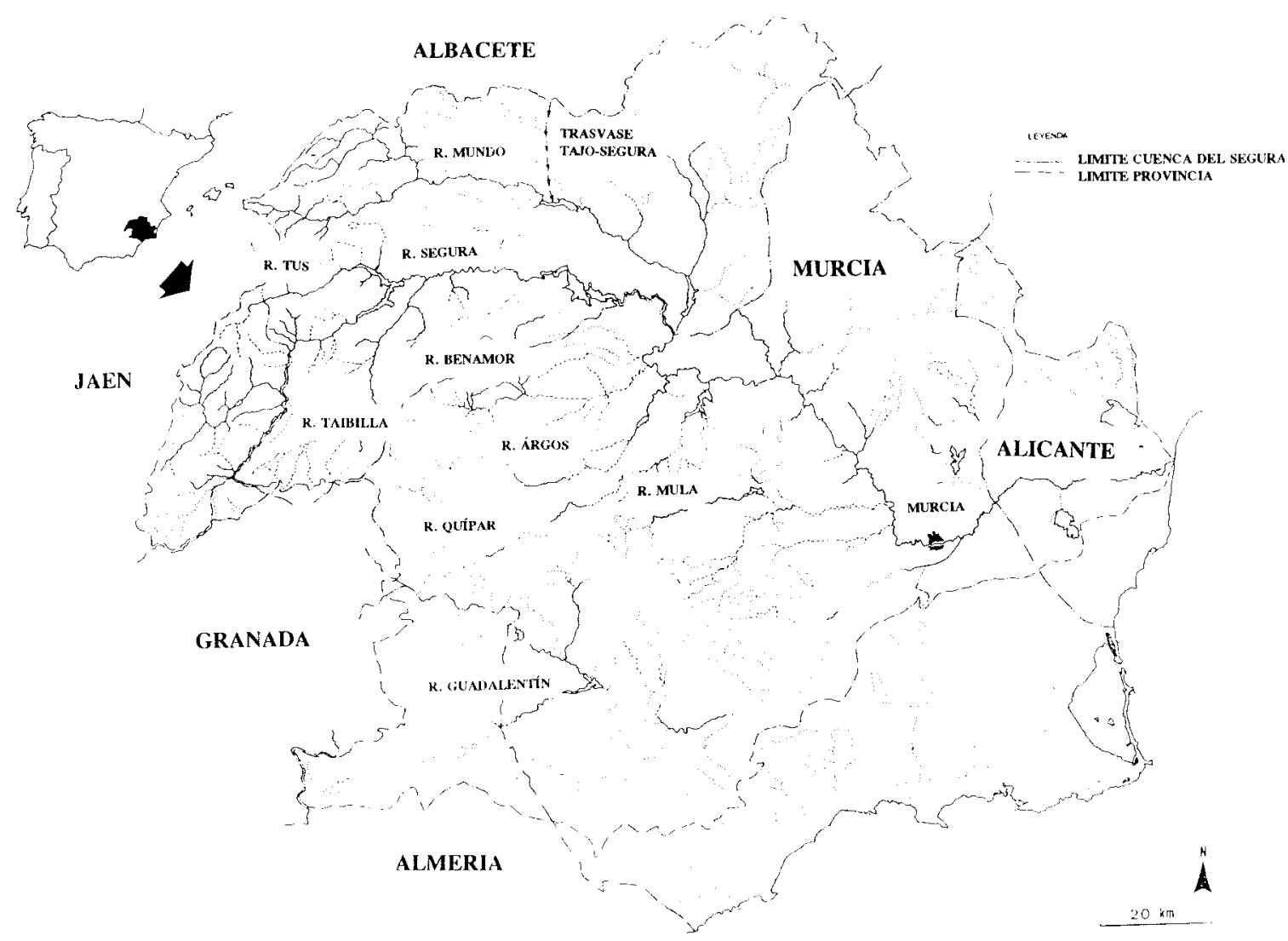

Figura Localización de la zona de estudio.

Figure I Location of the study area

Corrcspondencias (BENZECRI, 1973; HILL, 1979), para el análisis de datos noininales, y resulta muy útil en el tratainiento de matrices de presencia/ausencia (PALASON, 1990). Para llevar a cabo este análisis se utilizó el paquete estadístico SPAD.N (LERART et al., 1984).

Posteriormente, se elaboró una segunda matriz con las estaciones y las variables ambientales orderiadas en modalidades nominales u ordinalcs que se detallan cn la Tabla 1. A esta matriz, junto con la de presencia/ausencia ( $1 / 0)$, se les aplicó un análisis de Corrcspondencias Canónicas Múltiples (MCCA) mediante el programa CANOCO (TER BRAAK, 1987). Un tipo de técnica similar a esta, también con datos cualitativos, ha sido utilizada por LEBRETON et al. (1988), para macroinvertebrados acuáticos de forma satisfactoria.

A partir de las dos matrices anteriores. se coinplctó una tercera matriz de especies y modalidades de las variables ambientales (64×54), que refleja los datos de frecuencia de aparición de las especies cii cada una de las clases o rangos propuestos a modo de tabla de contingencia. Con el fin de equiparar las modalidades y especies por su presencia, se ponderó esta matriz, dividiendo cada columna (modalidades) por el número de veces que aparece dicha modalidad en la cuenca, y cada fila (especies) por el número dc estaciones de muestreo en las que aparece la especie en la zona de estudio. Arnbos casos se dan cri porcentajes (Apéndice 2). A la matriz resultante se le aplicó un análisis de correspondencias (CA), frecuentemente utilizado en el tratamiento de tablas de contingencia (BENZECRI. 1973). El paquete estadístico empleado en este análisis fue SPAD.N (LEBART et al., 1984).

\section{RESULTADOS}

\section{MCA: Análisis de Correspondencias Múltiple.}

En la Tabla 2 aparecen los autovalores o valores principales, inercia e inercia acumulada del análisis para los ejes I y II , así como las contribuciones absolutas para dichos ejes de las especies con valores màyores a 3 . 
Tabla 1. Rangos de los parámetros bióticos y abióticos medidos. " Rangos ordinales: o Rangos nominales. () Simbología de los rangos Table 1. Ranges of biotic and abiotic and abiotic parameters measured: * Numerical ranges o caregorical ranges. () Range codes.

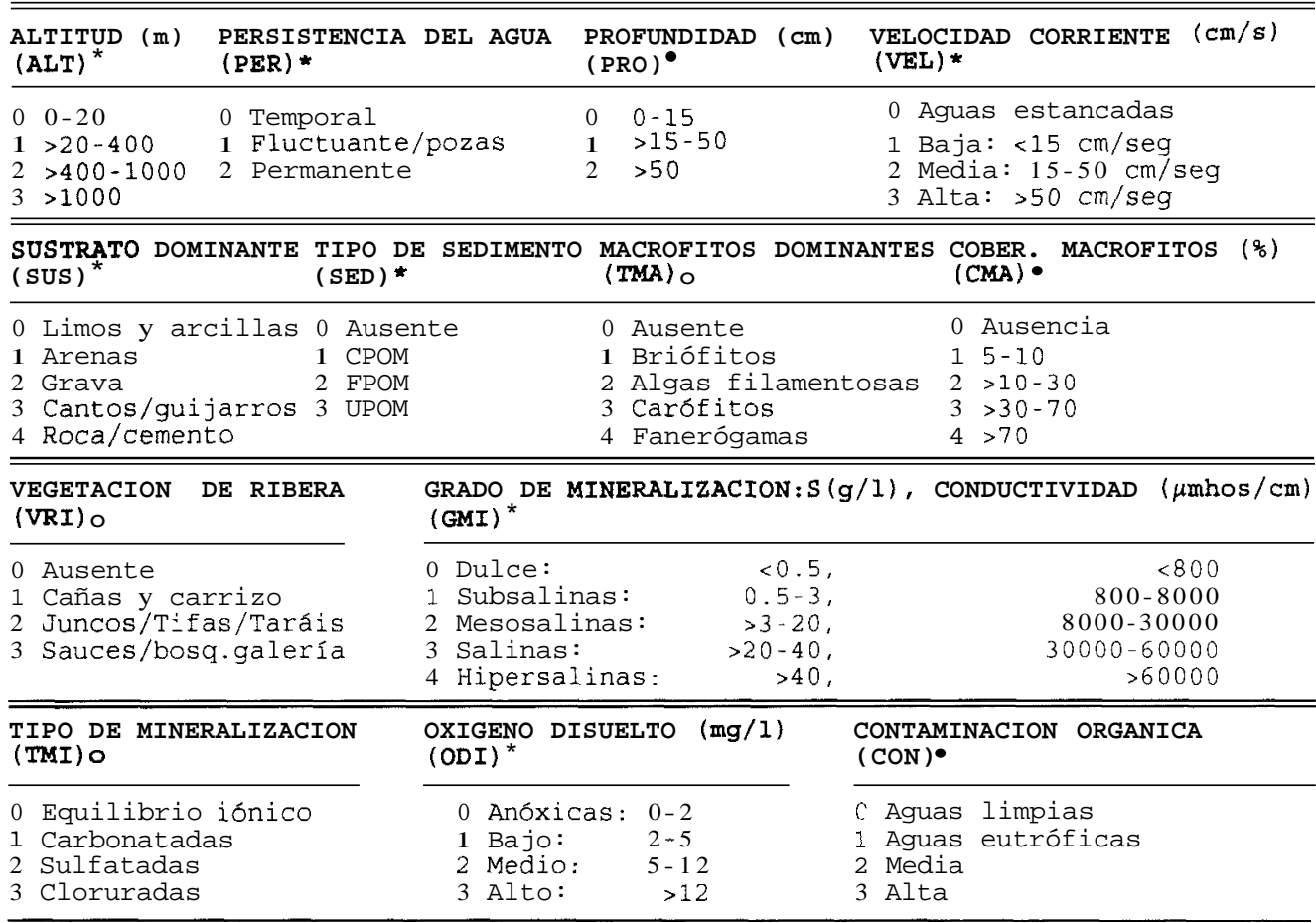

En la representación del plano factorial (Fig. 2) sc han seleccionado las especies rncjor representadas en el mismo, es decir, aquellas con una contribución relativa para la suma de los cjcs mayor de 0.1 (PALAZON, 1990). No se han considerado las ausencias de las especies, dado que a pesar de que algunas tienen contribuciones relativas altas, aparecen en el centro de las

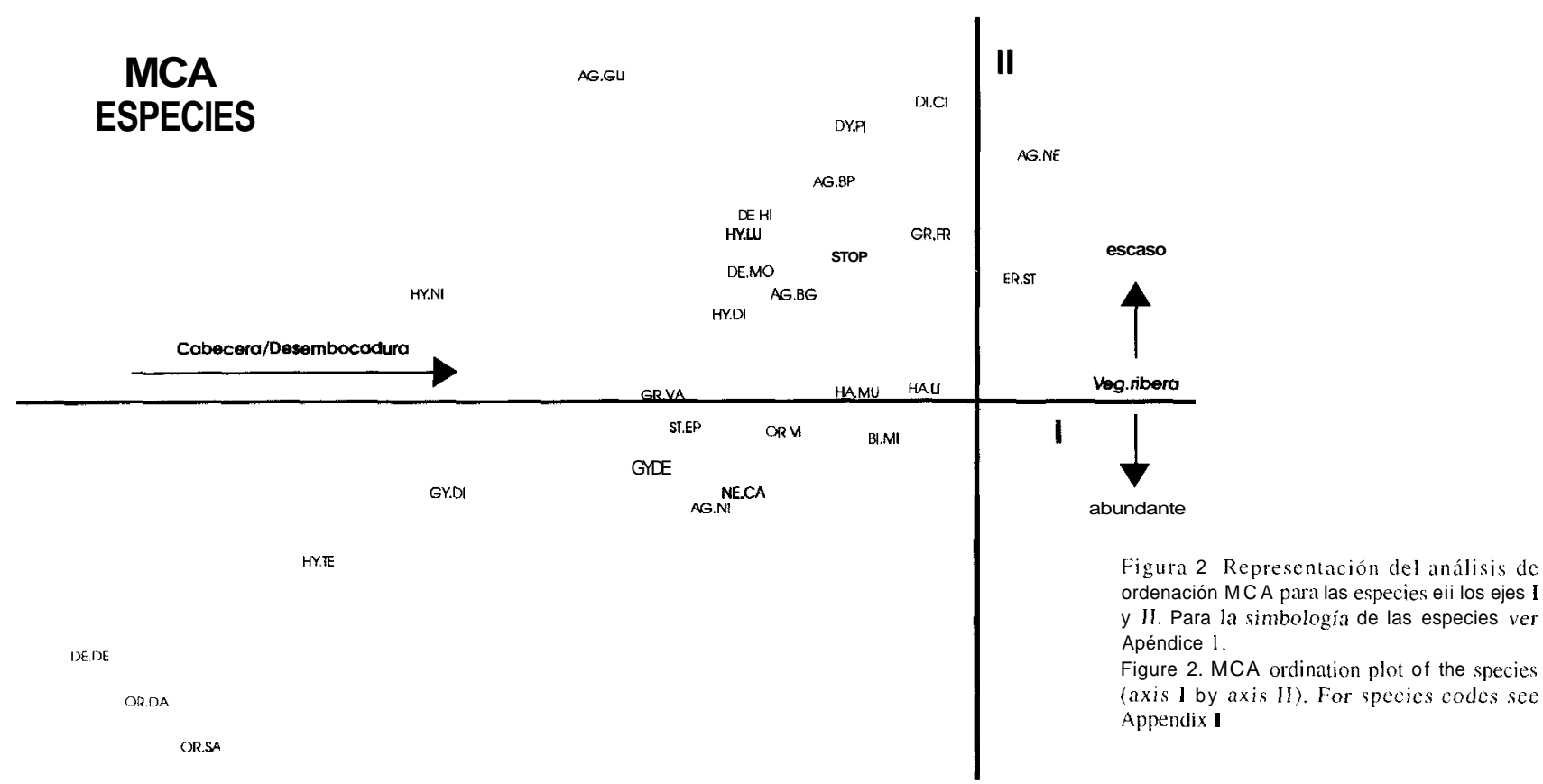


coordenadas o muy próximas a él y carecen de interés para la interpretación de los resultados. Tampoco se han representado las estaciones con objeto de no complicar la exposición gráfica.

A partir de la ordenación de las especies en el plano definido por los ejes I y II, se aprecia un gradientc desde la cabecera a la desembocadura de la cuenca definido por el eje I. Así, con valores riegativos aparecen, principalmente, especies como Gyrinus distinctus, G. dejeani, Orectochilus villosus, Hydroportss nigrita, H.tessellatus, H. discretus, Graptodytes varius, Stictonectes epipleuricus, Deronectes depressicollis, Nebrioporus $b$. cazorlensis y Oreodytes davisii, que se encuentran asociadas a estaciones de tramos altos de los ríos (por encima de los 1.000 in), con aguas dulces y velocidad de la comente elevada. En dirección a la parte positiva del eje, se observa un escalonainiento de especies hacia estaciones de los tramos inferiores del río.

El eje II. se relaciona con la presencia de vegetación de ribera, de modo que la parte positiva estaría representada por especies más propias de ambientes abiertos, con escasa vegetación y eutrofizados, corno Agabus bipustulatus, A. nebulosus o Eretes sticticus, y cn el extremo negativo aparecerían especies más propias de cuerpos de agua no eutrofizados, donde se conserva el bosque de galería.

MCCA: Análisis Canónico de Correspondencias Múltiple.

En la Tabla 3, aparecen los autovalores y varianza acumulada para los dos primeros ejes del MCCA, así como las correlaciones

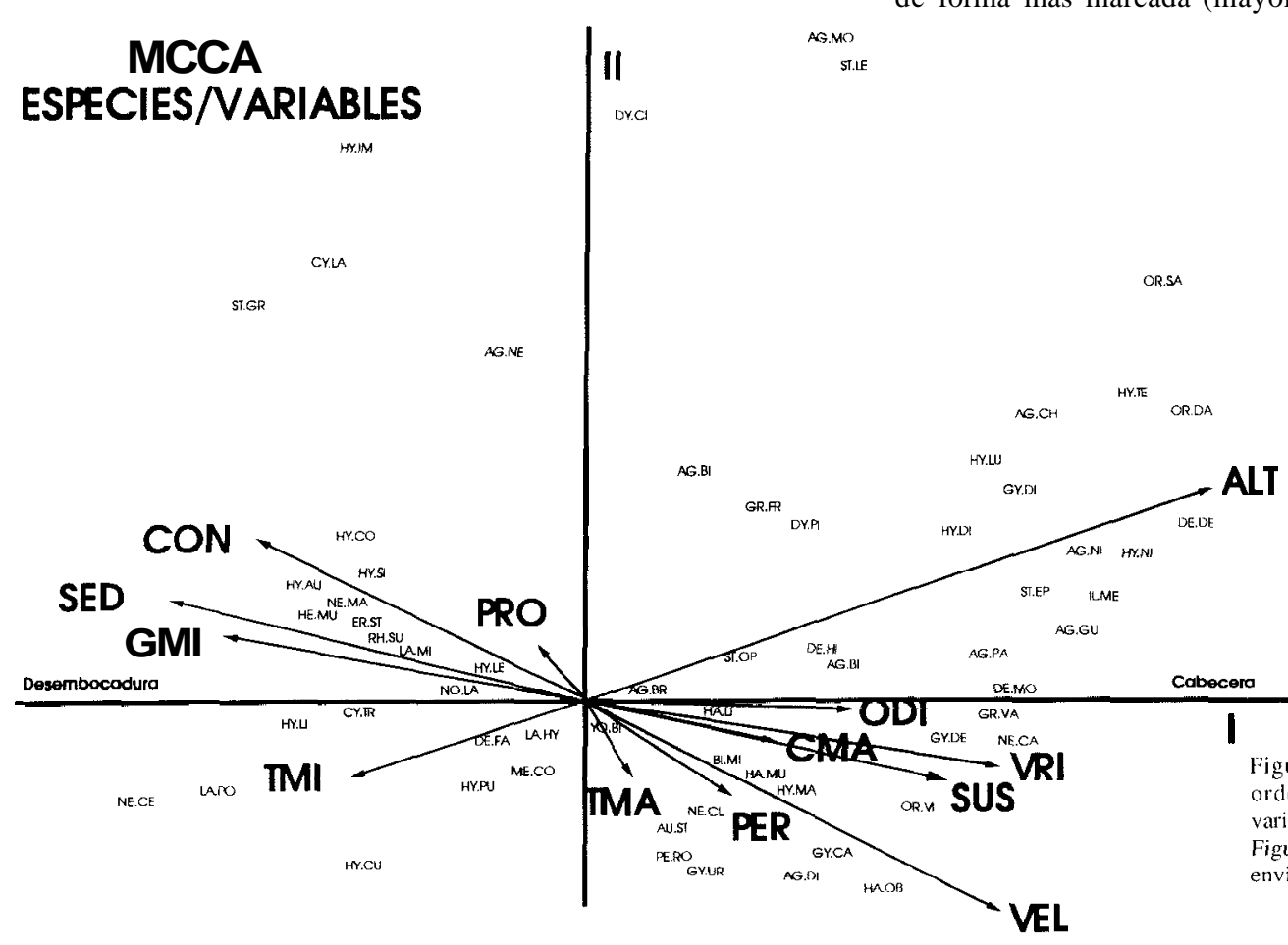

Tabla 2. Autovalores, inercia. inercia acumulada y contribuciones absolutas de las especies para los ejes I y II del MCA. Para la simbología dc las especies ver Apéndice 1. Table 2. Eigenvalues, inertia, conmulative inertia and absolute contribution of the specses for the axis I and Il of the MCA. For ihe species codes see Appendix I

\begin{tabular}{|c|c|c|}
\hline MCA & EJE I & EJE II \\
\hline AUTOVALORES . . . . & 0.81 & 0.50 \\
\hline $\begin{array}{l}\text { INERCIA } \\
\text { INERCIA ȦCUMUUL் } \dot{A} \dot{A}\end{array}$ & $\begin{array}{l}.8 .18 \\
\therefore 8.18\end{array}$ & $\begin{array}{r}5.01 \\
13.20\end{array}$ \\
\hline $\begin{array}{r}\text { CONTR . ABSOLUTAS } \\
\text { EJE I }\end{array}$ & & $\begin{array}{l}\text { SPECIES } \\
\text { EJE II }\end{array}$ \\
\hline GY.DI & AG . BP & 9.70 \\
\hline $\mathrm{HY} . \mathrm{NI}$ & HY . LU. & 7.00 \\
\hline OR.DA. & DY.PI. & 6.70 \\
\hline GY .DE & ST.OP & 6.20 \\
\hline HY .DI & AG.NE. & 5.40 \\
\hline GA.VA. & DE.KI. & 4.00 \\
\hline OR.VI. & DY.CI & 3.90 \\
\hline NE. CA & HY.DI & 3.80 \\
\hline HY. TE & OR.DA & 3.80 \\
\hline$\ldots 4.00$ & GR . GR & 3.70 \\
\hline$\ldots 3.90$ & ER.ST & 3.50 \\
\hline
\end{tabular}

y coeficientes canónicos de las variables ambientales para esos mismos ejes.

La distribución de las especies y variables en cl plano definido por los ejes I y II (Figura 3), sigue nuevamente, pero dc forma más marcada (mayor varianza acumulada para los 
Tabla 3. Aiitovalores. vamanza acumulada. correlaciones y coeficientes canónicos de las variables ambientales con los ejes I y II del MCCA.

Table 3. Eigenvalues, cumulative variance correlations and canonical coefficients of environmental variables for axis I and II of the MCCA.

\begin{tabular}{|c|c|c|}
\hline MCCA & EJE I & EJ II \\
\hline $\begin{array}{l}\text { AUTOV } \\
\text { VAR. }\end{array}$ & $\begin{array}{r}0.37 \\
.39 .10\end{array}$ & $\begin{array}{r}0.01 \\
51.00\end{array}$ \\
\hline \multicolumn{3}{|c|}{ CORRELACIONES } \\
\hline & EJE I & EJE II \\
\hline ALT. & 0.64 & 0.19 \\
\hline PER. & .0 .23 & -0.10 \\
\hline PRO. & -0.08 & 0.12 \\
\hline VEL , & .0 .43 & -0.29 \\
\hline SUS. & .0 .43 & -0.13 \\
\hline SED. & -0.48 & 0.13 \\
\hline TMA. & .0 .05 & -0.08 \\
\hline CMA. & .0 .18 & -0.02 \\
\hline VRI . & .0 .52 & -0.12 \\
\hline GMI & -0.46 & 0.11 \\
\hline TMI. & -0.26 & -0.07 \\
\hline ODI. & .0 .28 & -0.03 \\
\hline CON &.-0.34 & 0.14 \\
\hline \multicolumn{3}{|c|}{ COEFICIENTES CANONICOS } \\
\hline & EJE $I$ & EJE II \\
\hline ALT. & .0 .58 & 1.13 \\
\hline PER . & .0 .01 & -0.16 \\
\hline PRO. & .0 .11 & 0.27 \\
\hline VEL . & 0.19 & 0.50 \\
\hline SUS. & .0 .16 & -0.24 \\
\hline SED. & -0.20 & 0.05 \\
\hline TMA. & .0 .06 & -0.10 \\
\hline CMA. & 0.04 & 0.06 \\
\hline VRI. & .0 .24 & 0.02 \\
\hline GMI. & .0 .03 & 0.42 \\
\hline TMI. & .0 .00 & 0.15 \\
\hline ODI. & .0 .15 & 0.03 \\
\hline CON. & 0.09 & 0.32 \\
\hline
\end{tabular}

dos primeros ejes). un gradiente desde la cabecera a la deseinbocadura. Así, el eje I se interpreta como el "eje" real de la cucnca del río Segura, de manera que la mayoría de las variables ambientales se distribuyen próximas a él, situándose en los extremos aquellas que son clarainente representativas de la cabecera (coordenadas positivas), como la altitud, vegetación de ribera en galería, sustrato grueso, velocidad de corriente alta y elevado contenido en oxígeno disuelto; o de la descmbocadiira (coordenadas negativas), corno alto contenido cn sediincnto orgánico, aguas muy mineralizadas y cloruradas, y contaminación orgánica elevada

Próxiinas al centro de coordenadas se encontrarían las variables que explican situaciones más dináinicas y fluctuantes en determinadas zonas de la cucnca, como pueden ser la profundidad y persistencia del agua o la menor prcscncia de vegetación de ribera propias, sobre todo, de arroyos de la vega media.

Las especies también sc distribuyen, en general, a lo largo del eje I, siendo las de la cabecera, las mencionadas para el MCA más Oreodytes sanmarkii, y Agabus nitidus, mientras que las de la vega baja y desembocadura son principalmente, Nebrioporus ceresyi, Laccophilus ponticus, Hydroporus limbatus, Hyphydrus aubei, Herophydrus musicus, Hydroglyphus signatellus, Nebrioporus baeticus e Hygrotus confluens.

Tabla 4. Aiitovalores, inercia, inercia acunulada y contribuciones absolutas de las especies y variables ambientales para los ejes I y 11 del CA.

Table 4. Egeuvalves, inertia, cumulative inertia and absoliite contributions of species and environmental variables for the I nnd Il axis of the CA

\begin{tabular}{|c|c|c|}
\hline CA & EJE I & EJE \\
\hline AUTOVALORES . & 0.17 & \\
\hline INERCIA . . . . . . . & 0.43 & \\
\hline INERCIA ACUMULAD & 0.43 & \\
\hline
\end{tabular}

CONTRIBUCIONES ABSOLUTAS

\begin{tabular}{|c|c|c|c|}
\hline EJE I & ESPECIES & & MODALIDADES \\
\hline $\begin{array}{l}\text { HA. LI. } \\
\text { HE . MU. } \\
\text { NO . LA. } \\
\text { DY . CI. } \\
\text { HY .MA. } \\
\text { AG.DI. } \\
\text { RH. SU. }\end{array}$ & $\begin{array}{r}13.94 \\
8.69 \\
8.02 \\
5.31 \\
5.00 \\
4.89 \\
3.89\end{array}$ & $\begin{array}{l}\text { ODI1 } \\
\text { ALTO } \\
\text { GM13 } \\
\text { ALT3 } \\
\text { GMI2 } \\
\text { GMI4 } \\
\text { SED1 } \\
\text { CON2 } \\
\text { VRI1 } \\
\text { VR13 } \\
\text { TM13 }\end{array}$ & $\begin{array}{cc}\ldots \ldots & 11.26 \\
\ldots \ldots & 8.30 \\
\ldots \ldots & 6.97 \\
\ldots \ldots & 6.64 \\
\ldots \ldots & 6.05 \\
\ldots \ldots & 5.96 \\
\ldots \ldots & 5.41 \\
\ldots \ldots & 5.09 \\
\ldots \ldots & 4.93 \\
\ldots \ldots & 3.87 \\
\ldots \ldots & 3.79\end{array}$ \\
\hline EJE II & ESPECIES & & MODALIDADES \\
\hline $\begin{array}{l}\text { RH. SU } \\
\text { AG.DI } \\
\text { HE. MU } \\
\text { HA. . II } \\
\text { DY.CI } \\
H Y . M A\end{array}$ & $\begin{array}{lr}\ldots \ldots & 23.95 \\
\ldots \ldots & 20.44 \\
\ldots \ldots & 19.53 \\
\ldots \ldots & 16.87 \\
\ldots \ldots & 8.16 \\
\ldots \ldots & 5.31\end{array}$ & $\begin{array}{l}\text { ODI1 } \\
\text { GM13 } \\
\text { GM14 } \\
\text { ALTO } \\
\text { SED2 }\end{array}$ & $\begin{array}{ll}\ldots \ldots & 52.28 \\
\ldots \ldots & 11.51 \\
\ldots \ldots & 10.66 \\
\ldots \ldots & 5.20 \\
\ldots \ldots & 4.57\end{array}$ \\
\hline
\end{tabular}

Por el contrario, las especies próxiinas al centro de coordenadas son, cn general, más eurioicas, con una amplia distribución en la cuenca. Tal es el caso de Laccophylus hyalinus, Yola bicarinata, Hydroglyphus pusillus, Agabus brunneus, Meladema coriacea, Hydaticus leander y Noterus laevis, con una cierta tendencia a la parte baja de la cucnca, y Agabus bipustulatus, Aulonogyrus striatus, Gyrinus urinator, Nebrioporus clarki, Haliplus lineatocollis, Peltodytes rotundatus y Bidessus minutissimus, con preferencia por la parte alta de la cuenca. 
Aquellas especies que aparecen aisladas y lejos del eje I, son raras o muy escasas en la cuenca del río Segura, reflejando únicamente situaciones puntuales. Este es el caso de Stictotarsus griseostriatus (cf. griseostriatus según NILSSON \& HOLMEN, 1995), Hygrotus impressopunctatus, Cybister lateralimarginalis o Hydrovatus cuspidatus en la vega baja y desembocadura, y de Dytiscus circumflexus, Agabus montanus, Stictonectes lepidus o Haliplus obliquus en los tramos de cabecera.

Este último aspecto sirve de interpretación al eje II, el cual, probablemente explica circunstancias particulares debido al carácter esporádico de algunas especies. Por otra parte, las bajas correlaciones de las variables ambientales con este eje ponen de manifiesto su escaso interés para la interpretación de la estructura de la comunidad.

Todo ello hace pensar en la existencia de un Único y bien definido gradiente altitudinal, asociado a unas características inicroambicntales propias, que se refleja, no sólo en la comunidad de Adephaga acuáticos, sino en la práctica totalidad de las variables muestreadas.

\section{CA: Análisis de Correspondencias}

La Tabla 4 muestra, como resultado del CA, los autovalores. inercia e inercia acumulada de los ejes I y II, y las contribuciones absolutas de las especies y modalidades de los parámetros ambientales iguales o mayores a 3 , a dichos ejes.

La representación del diagrama de ordenación en los distintos planos factoriales se ha realizado, con las coordenadas de las especies y modalidades cuya suma de las contribuciones relativas para los ejes 1 y II fuera igual o superior a 0.1 (PALAZON, 1990). Para una visión más clara de los resultados, la distribución espacial de las especies y las modalidades aparecen en gráficos separados (Figs. 4 y 5).

Los diagramas de ordenación para especies y modalidades en ejes los I y II, corroboran y mejoran los resultados obtenidos anteriormente, en los que el primer eje, con una altísima inercia del $43 \%$ (Tabla 4) explican la distribución de las especies desde los tramos altos (extremo negativo) de la cuenca del Segura hasta la desembocadura (extremo positivo). Así, se encuentra en la parte positiva del eje de coordenadas,

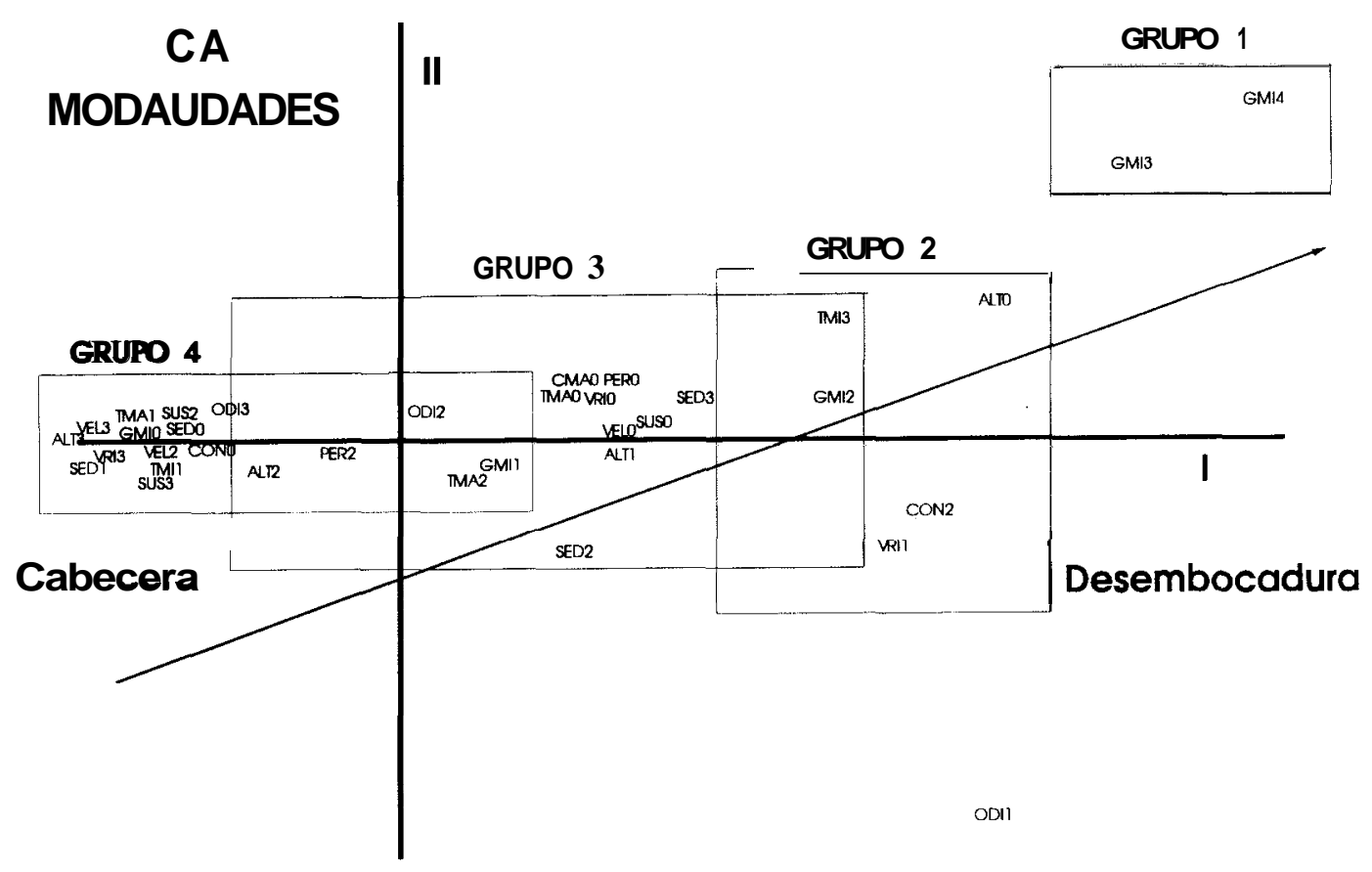

Figura 4 Representación del análisis de ordenación CA para los rangos de las variables ambientales en los ejes l y 11 Figure 4 CA ordination plot of range ot environmental variables (axıs I by axis II). 


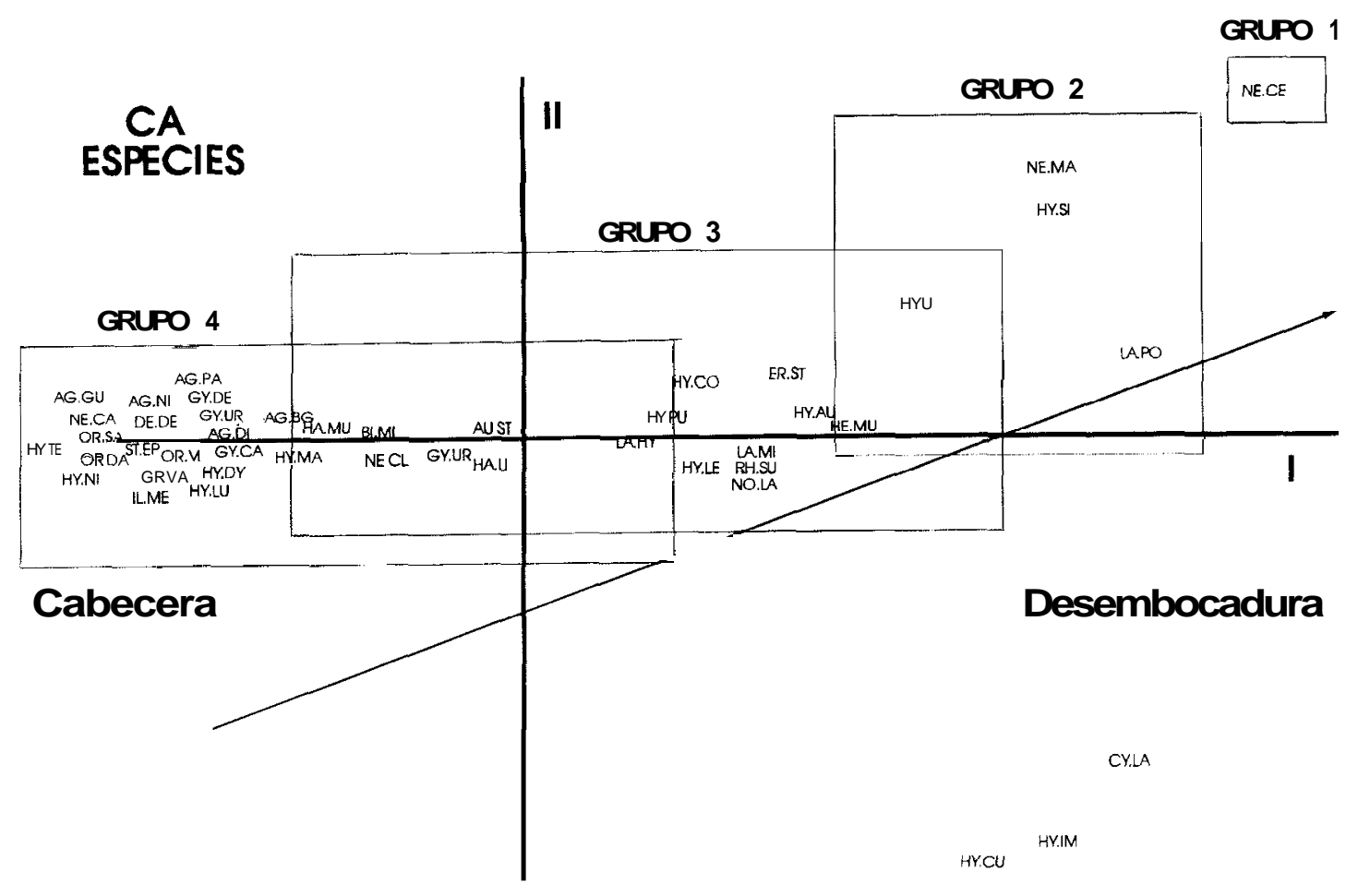

Figura 5. Representación del análisis de ordenación CA para las especies en los ejes I y II

Figure 5. CA ordination plot of the species (axis I by axis 11).

especies como Herophydrus musicus, Noterus laevis o Rhantus suturalis, y modalidades como oxígeno disuelto 1 , altitud 0 , grado de mineralización 2, 3 y 4, contaminación orgánica 2, vegetación de ribera 1 y tipo de mineralización 0 (ver Tabla 1), mientras que en su parte negativa, las especies que contribuyen con un mayor peso a su formación son Haliplus lineatocollis, Hydroporus marginatus o Agabus didymus, y modalidades altitud 3, tipo de sedimento 1 , vegetación de ribera 3, tipo de rnacrófitos 1 y grado de mineralización 0 .

La ventaja de este análisis, ha sido la posibilidad de asociar con mayor claridad, las preferencias de algunas especies o grupos de especies por determinados rangos dc las variables del medio. El resultado final ha sido la caracterización de 4 grupos ecológicos, bien definidos, dentro del gradiente general observado en la cuenca:

Grupo 1: formado únicamente por Nebrioporus ceresyi, que es exclusivo de aguas salinas e hipersalinas.

Grupo 2: constiiuido por Laccophilus ponticus, Hydroglyphus signatellus y Nebrioporus baeticus los cuáles se encuentran. prefcrentementc, en cursos de agua en tramos bajos de la cuenca de carácter, mesosalinos o salinos, con un contenido orgánico elevado (eutrofizadas o muy contaminadas) y vegetación de ribera escasa (cañas o carrizo).
Hydroporus limbatus y Herophydrus musicus. actuarían a modo de "especies puente", entre este grupo y el siguiente.

Grupo 3: formado por Laccophilus minutus, Hygrotus confluens, Hyphydrus aubei, Rhantus suturalis, Hydaticus leander, Eretes sticticus y Noterus laevis, presentes con mayor frecuencia en aguas temporales o muy fluctuantes, con abundante sedimento y materia orgánica finamente particulada, pero menos mineralizadas $y$, en general, con mayor contenido en oxígeno disuelto que el grupo 2, aunque para este último factor, el rango de variación encontrado ha sido muy amplio. La vegetación de ribera de estos ambientes está ausente o es de bajo porte (cañas, carrizo o juncos).

Grupo 4: es el más numeroso y está formado, principalmente, por Hydroporus nigrita, H. discretus, H. marginatus, H. lucasi, Oreodytes davisii, 0 . sanmarkii, Gyrinus caspius, $G$. dejeani, $G$. distinctus, Orectochilus villosus, Nebrioporus b cazorlensis, Stictonectes epipleuricus, Deronectes depressicollis, B. moestus, Agabus didymus, A. paludosus, A. nitidus, A. biguttatus, A.guttatus e lliybius meridionalis. Son, en general, especies que viven en ambientes por encima de los $500 \mathrm{~m}$, de aguas corrientes (aunque ocupen los remansos), limpias y bien oxigenadas, dulces y permanentes, sustrato grueso, con musgos y abundante vegetación de ribera que forma bosque de galería. 
Por último. Laccophylus hyalinus, Haliplus lineatocollis, $H$. mucronatus, Hydroglyphus pusillus, Bidessus minutissimus, Aulonogyrus striatus, Gyrinus urinator y Nebrioporus clarki, tienen una amplia distribución en la cuenca del Segura, por lo que también se pueden considerar especies de características interrnedias o puente entre los dos últimos.

\section{DISCUSIÓN}

Actualmente, la técnica de ordenación rnás utilizada para relacionar especies de macroinvertebrados y hábitats (estaciones de rnucstreo) es la de DECORANA (Dctrented Correspondence Analysis -HILL, 1979; FURSE et al., 1984; ARMITAGE et al., 1990; FOSTER et al., 1992; EYRE et al.. 1993; COLLINSON et al., 1995; CLARK \& SAMWAYS, 1996). de la que los MCA y CA aplicados en este estudio son rneras variantes. Sin embargo, para asociar especies y variables ambientales en el espacio, CANOCO (Canonical Correspondence Analysis TEER BRAAK, 1987) es también una técnica válida ya que calcula las correlaciones de los ejes de las especies con las variables ambientales, a pesar de que no son muchos los estudios que aplican esta técnica (p.ej. LEBRETON et al., 1988 para rnacroinvertebrados; GACIA et al., 1994 para inacrófitos; SANDERSON et al., 1995 para invertebrados), quizá debido a una mayor dificultad en la interpretación de los resultados del análisis.

En cste trabajo. el empleo de CANOCO (MCCA) ha reflejado tendencias de distribución de los colcópteros acuáticos muy sirnilares a las obtenidas con MCA y CA, siendo más precisas, incluso, que para el MCA. Sólo la utilización de matrices de datos más elaboradas, como en el caso de la tabla de contingencia para el CA, ha permitido determinar. de forma más evidente, grupos de especies asociados a rangos o "inodalidades" de las variables del medio inás concretos (ver tabla 1).

Así, aunque cualquiera de las técnicas de ordenación usadas han dado resultados parecidos, se puede concluir que los patrones de distribución general de las comunidades de coleópteros Adephaga acuáticos, han quedado mejor definidos con MCCA $\mathrm{y}$, sobre todo con $\mathrm{CA}$. con una varianza e inercia acumulada para los dos primeros ejes muy alta, $51 \%$ y $54 \%$ respectivamente. De este modo, datos de frecuencia de aparición de las especies en estaciones cuyas variables ambientales han sido previamente clasificadas cn rangos se han revelado de grari valor cara al estudio de la distribución de cste grupo de organismos.

De los factores del medio estudiados, la altitud, el grado de mineralización y la presencia y tipo de vegetación de ribera, son los que mejor explican la distribución de los Adephaga acuáticos en la cuenca del Segura. Otros autores corno GARRIDO et al., (1994) y RIBERA et al., (1996). también destacan la importancia de la altitud y mineralización respectivamente, en la distribución de gran número de especies de coleópteros acuáticos. La altitud, originaría un gradiente ambiental irnportante en un espacio rclativarnente pequeño, limitando la colonización de determinadas especies, sobre todo en zonas elevadas, donde los parámetros asociados a la misma, actuarían como factor limitante.

La inineralización sería el condicionantc que controlaría la colonización de gran parte de las especies en la zona de la vega media-baja, dado que la mayoría de los cuerpos de agua de la zona de estudio son de carácter subsalino a hipersalino debido al tipo de sustrato y a las características climáticas. La vegetación de ribera juega un papel muy importante a nivel estructural (soporte para las puestas, generador de refugios, organizador de microambientcs donde viven otras especies que sirven de alimento y, como recurso alimenticio, si consideramos su descomposición). Así, su efecto regulador en la distribución de los Adephaga acuáticos es claro por tanto, viéndose incrementada su iinportancia en la zona de estudio por las marcadas diferencias ambientales y antropomórficas de los cauces entre cabecera y desembocadura (GONZALEZ DEL TANAGO et al., 1995).

Por el contrario, la elevada profundidad del cuerpo dc agua y el grado de cobertura de macrófitos, son los parámetros que menos importancia tienen. El bajo número de muestreos realizado en ambientes de aguas profundas, por su escasez o por su inaccesibilidad (las balsas de riego están cercadas en su mayoría) y el poco interés que la mayoría de los Adephaga acuáticos presentan por estos medios podría justificar el escaso papel regulador que juega la profundidad. Sólo algunas especies grandes de los géneros Cybister y Eretes tienen cierta preferencia por cuerpos de agua de grandes dimensiones y profundos.

Los macrófitos, parecen tener más iinportancia a nivel cualitativo que cuantitativo. Así, la presencia de microambientcs generados por inacrófitos y la propia vegetación de ribera, que sirvan como fuente de alimento y refugio, sería más significativo que la extensión o repetición de tales rnicroambientes.

Otro aspecto a resaltar es el gradiente biológico detectado en contraste con la heterogeneidad ambierital de la cuenca del Segura (VIDAL-ABARCA et al., 1992). La alta capacidad de dispersión y el carácter oportunista de algunas de las especies estudiadas (Haliplus lineatocollis, Laccophilus hyalinus o Herophydrus musicus), les permitiría colonizar la mayoría de cuerpos de agua estudiados, uniformizando dichos ambientes $\mathrm{o}$, al menos poniendo de manifiesto aspectos comunes en 
hábitats aparentemente diferentes. Por el contrario, la presencia de determinadas especies, como Deronectes depressicollis, Oreodytes davisii y $O$. sanmarkii, o Nebrioporus baeticus y $N$. ceresyi, más especialistas, y con una tiistribucióri más restringida cn relación al eje cabecera desembocadura, ha permitido delimitar cuatro grupos cenológicos bien definidos, uno típico de cabecera (grupo 4), dos de ambientes más mineralizados y antropomorfizados (grupos I y 2) y finalmente un grupo de hábitats más heterogéneos. Aunque estos resultados se enmarcan en un ámbito regional. la existencia dc asociaciones de especies similares a los detectadas en otras cuencas, sin que existan marcadas diferencias a nivel biogcográfico, haría factible extrapolar condiciones climáticas, ecológicas y de azar parecidas a dichas áreas. Del mismo modo, determinadas condiciones ambientales semejantes a las detectadas en la cuenca del Segura, posibilitarían la presencia de grupos cenológicos inuy próximos a los encontrados en la zona de estudio.

Así, el valor indicador de los coleópteros acuáticos sería reflejo, no sólo de las condiciones presentes de la inayor parte de hábitats, sino de factores históricos que han condicionado su presencia en los mismos, lo que redundaría más en su interés. Este interés no lleva implícito su utilización en todos los estudios, pero sí refleja la necesidad de tenerlos en cuenta en aquellos ambientes donde son el grupo mayoritario (RIBERA \& FOSTER, 1992).

\section{AGRADECIMIENTOS}

Queremos expresar nuestro agradecimiento a José Fraiicisco Calvo Sendín y a José Antonio Palazón Ferrando por su ayuda cn el tratamiento estadístico de los datos.

Asimismo. los comentarios y sugerencias dc Ignacio Ribera nos han servido de gran utilidad.

\section{BIBLIOGRAFÍA}

ARMITAGE. P.D.; I. PARDO: M.T. FURSE \& J.F. WRIGHT. 1990. Assessment and prediction of biological quality. A demostration of a British macroinvertebratebased inethod in two Spanish rivers. Limnética, 6: 147-156.

RAGGE, P. 1983. The macrobenthos of the River Tourujoki and its tributaries (Central Finland). 2, Odonata. Heteroptera and Coleoptcra. Acta Entomol. Fenn., 42: 15-22.
BENZECRI, J.P. 1973. L'analyse des données, II. L'analyse des correspondances. Dunod. Paris.

BIESIADKA, E. 1980. Water beetles (Coleoptera) of the eutrofic Lakc Zbechy (Leszno Voiv.). Polish Ecological Studies, 6(2): 263-275.

ROURASSA, J.P.; Y. ALARIE. \& R.Jr. LECLAIR. 1986. Distribution and habitat selection of Dytiscid Beetles in characteristic vegetal units of southern Québec. Entomol. Basil., 11: 289-296.

BRANCUCCI, M. 1980. Observations on the ccology of the Dytiscidae (Colcoptera) in the watcr system connected to the south coast of Neuchatel lake (Switzcrland). Mirr. Schweiz. Entomol. Ges. 53(4): 365-78.

BUSSLER, H. 1988. Zwcitcr Beitrag zur Dytisciden und Hydrophilidenfauna Nordbayers (Col., Dytiscidac. Hydrophilidae). Nachr: Bayer Entomol., 37(1): 5-10.

CLARK, T.E \& M.J. SAMWAYS. 1996. Dragoriflies (Odonata) as indicators of hiotipc quality in ihe Kruger National Park. South Africa. .1.Appl. Ecol., 33: 1001-1012.

COLLINSON, N.H.; J. BIGGS; A. CORFIELD; M.J. HODSON; D. WALKER; M. WHITFIELD \& P.J. WILLIAMS. 1995. Temporary and permanent ponds: an assessment of the effects of drying out on the conservation valuc of aquatic inacroinvertebrate communities. Biol. Conserv., 74: 125-133.

EYRE, M.D.; S.G. HALL \& G.N. FOSTER. 1986. A initial classification of the habitats of aquatic Coleoptera in NorthEast England. J. Appl. Ecol., 23(3): 841-852.

EYRE, M.D. \& G.N. FOSTER. 1989. A comparison of aquatic Heteroptcrn and Coleoptcra coinmunities as a basis Sor cnvironmental and conservation assesments in static water sites. J. Appl. Ent., 108: 355-362.

EYRE, M. D. \& S. P. RUSHTON, 1989. Quantification oí conservation criteria using invertebrates. J. Appl. Ecol., 26: 159-71.

EYRE, M.D.; J.G. PILKINGTON; R. CARR; R.P. McBLANE; S.P. RUSHTON \& G.N. FOSTER. 1993. Thc running-water beetles (Coleoptera) of a rivcr catchinciit in northern England. Hydrobiol., 264: 33-45.

FOSTER, G.N.; A.P. FOSTER. M.D. EYRE. \& T. BILTON. 1990. Classification of watcr beetle assemblages in arable fenland and ranking of sites in relation to conservation valuc. Freshwat. Biol., 22: 343-354.

FOSTER. G.N.: B.H. NELSON; D.T. BILTON; D.A. LOTT; R. MERRIT; R.S. WEYL \& M.D. EYRE. 1992. A classification and cvaluation oí Irish watcr beetles assemblages. Aquatic Conservation, 2: 185-208.

FRIDAY, L.E. 1987. The divcrsity of macroinvertebrate and 
macrophyte communities in ponds. Freshwat. Biol., 18: 87-104.

FURSE, M.T.; D. MOSS; J.F. WRIGHT \& P.D. ARMITAGE. 1984. The influence of seasonal and taxonomic factors on the ordination and classification of running-water sites in Great Britain and on the prediction of their macroinvertebrate communities. Freshwat. Biol., 14: 257-280.

GACIA. E.; E. BALLESTEROS; L. CAMARERO; O. DELGADO; A. PALAU; J.L. RIERA \& J. CATALAN. 1994. Macrophytes from lakes in the eastern Pyrenees: community composition and ordination in relation to environmental factors. Freshwat. Biol., 32: 73-81.

GARRIDO, J.; M. FERNANDEZ \& J.A. REGIL. 1994. Geographical distribution of Adephaga and Polyphaga (Coleoptera) in the Cantabrian Mountains (Spain): Specific richness and analysis of the altitude factor. Arch. Hydrobiol., 131 (3): 353-380.

GONZALEZ DEL TANAGO, M.; M.R. VIDAL-ABARCA: M.L. SUAREZ \& C. MOLINA. 1995. Consideraciones sobre el estado de las riberas de los principales cauces fluviales de la cuenca del río Segura (S.E. de España). An. Biol., 20(9): 117-130.

HANSON, B. A. \& G.A. SWANSON. 1989. Coleoptera species inhabiting prairie wetlands of the Cottonwood Lake area, Stutsman County, North Dakota. Prairie Nat., 21 (1): 49-57.

HEUSS, K. 1989. Water beetles as indicators of water quality. Balfour-Browne Club, Newsletter, 44: 7-12.

HILL, M.O. 1979. DECORANA-A FORTRAN program for detrented correspondence analysis and reciprocal averaging. Ecology and Systematics, Cornell University, New York.

HOSSEINIE, S.O. 1978. Aquatic Coleoptcra from Southwestem Iran (Haliplidae, Dytisciade and Gyrinidae, with a note on Hydrophilidae). The Coleopterists Bull., 32(2): 167-175.

JEFFRIES, M. 1988. Do water beetles communities reflect the wider freshwater community? The Balfour-Browne Club. Newsletter, 42: 14-17.

LANCASTER. J. \& G.G.E. SCUDDER. 1987. Aquatic Colcoptera and Hemiptera in some Canadian saline lakes: patterns in community structure. Can. J. Zool., 65: 1383-90.

LARSON, D.J. 1985. Structure in Temperate predaceus diving beetle communities (Coleoptera Dytiscidae). Holartic Ecol., 8(1): 18-32.

LEBART, L.; A. MORINEAU. \& K.W. WARWICH. 1984. Multivariate descriptive stadistical analysis. Correspondence analysis and related techniques for large matrices. Wiley. New York.

LEBRETON, J.D.; D. CHESSEL \& M. RICHARDOT. 1988. L'analyse des relations especes-milicu per l'analyse canonique des correspondences. HI. Variables de milieu qualitatíves. Acta Oecologica, Oecol. Gener, 9(2): 137-151.

MIELEWCZYK, S. 1983. Quantitative investigations on Odonata, Heteroptera and Coleoptera in a drainage channel near the village of Turew (Poznan region). Acta Hydrobiol., I(25-26): 89-100.

MILLAN, A. 1991. Los Coleópteros Hydradephaga (Haliplidae, Gyrinidae, Noteridae y Dytiscidae) de la cuenca del rio Segura, SE de la Península lbérica. Tesis Doctoral. Universidad de Murcia.

MILLAN, A; J. VELASCO \& A.G. SOLER. 1992. Los coleópteros Hydradephaga de la cuenca del río Segura (SE de la Península Ibérica). Aspectos faunísticos más relcvantes. An. Biol., 18(7): 39-45.

MONTES, C.; L. RAMIREZ-DIAZ \& A.G. SOLER. 1980. Las taxocenosis de colcópteros acuáticos como indicadores ecológicos en el río Rivera de Huelva (Sevilla): Aspectos metodológicos. An. Univ. Murcia (Ciencias), 33: 23-40.

MORENO, J.L.; A. MILLAN; M.L. SUAREZ; M.R. VIDALABARCA \& J. VELASCO. 1997. Aquatic Coleoptera and Heteroptera assemblages in waterbodies from ephemeral coastal streamus ("ramblas") of south-eastern Spain. Arch. Hidrobiol., 141(1): 93-107.

NILSSON. A.N \& M. HOLMEN. 1995. The aquatic Adephaga (Coleoptera) of Fennoscandia and Denmark. II. Dytiscidae. Fauna Entomologica Scandinavica, Vol. 32. E.J. Brill. Leiden.

PALAZON, J.A. 1990. Análisis de Correspondencias Múltiples en Ecologia Descriptiva: Métodos, Programas y Aplicaciones. Tesis Doctoral. Universidad de Murcia.

RIBERA, I. \& G.N. FOSTER. 1992. Uso de los coleópteros acuáticos como indicadores biológicos (Coleoptera). Elytron, 6: 61-75.

RIBERA, I.; J. ISART \& J.A. REGIL. 1995 a. Autoecología de algunas especies de Hydradephaga (Coleoptera) de los Pirineos. I. Gyrinidae, Halíplidae, Noteridae e Higrobidae. Zool. Baetica, 7: 33-58.

RIBERA, I.; J. ISART \& J.A. REGIL. 1995b. Autoecología de algunas especies de Hydradephaga (Coleoptera) de los Pirineos. II. Dytiscidae. Zool. baetica, 6: 59-104.

RIBERA, I.; D.T. BILTON; P. AGUILERA \& G.N. FOSTER. 1996. A North African-European transition fauna: water beetles (Coleoptera) from the Ebro delta and other Mediterranean coastal wetlands in the Iberian peninsula. Aquatic Conservation, 6: 121-140.

RICHOUX, P. 1988. Inventaire des Coléoptères des cours d'eau: comparaison de méthodes de prélèvement. Naturaliste Can., 115: 223-228. 
RICHOUX. P. \& E. CASTELlA. 1986. The aquatic Coleoptera of former riverbeds submitted to large hydrological fluctuations. Proc. 3rd European Congr. Entomol. (Amsterdan, 24-29 August): 129-32.

SOLER, A.G. 1972. Los coleópteros acuáticos de las marismas riel Bajo Guadalquivir. Publ. Univ. Sevilla. 42 pp.

SANDERSON. R.A.; S.P. RUSHTON; A.J. CHERRILL \& J.P. NYRNE. 1995. Soil, vegation and space: an analysis of their effects on the invertebrate coinmunities of a moorland in north-east England. J. Appl. Ecol., 32: 506-518.

TER BRAAK. C.J.F. 1987. CANOCO - a FORTRAN program for canonical community ordination by [partial] [detrended] [canonical] correspondence analysis, principal components analysis and redundance analysis (version 2,1). Agricultura1 Mathematics Groups. Wagcningen.

VIDAL-ABARCA. M.R. 1985. Las aguas stiperficiales de la cuenca del río Segura (SE. de España). Caracterización fisico-quimica en relación al medio físico y humano. Tesis doctoral. Universidad de Murcia.

VIDAL-ABARCA, M.R.; M.L. SUAREZ \& L. RAMIREZ-DIAZ. 1992. Ecology of Spanish semiarid streams. Limnética, 8: 151-160. 
Apéndice 1. Adephaga acuáticos de la cuenca del río Segura. "Especie encontrada después del análisis de los datos. ": Especic en revisión. considerada en el análisis de los datos como A. brimneris.

Appendix 1. Aquatic Adephaga from Segura River Basin. "Species recorded after the data analysis. ${ }^{*}:$ Species under review. For the data analysis it was considered A. brumnells.

\begin{tabular}{|c|c|}
\hline ADEPHAGA ACUATICOS DE LA CUENCA DEL SEGURA & SIMBOLOGIA \\
\hline $\begin{array}{ll}\text { F. Gyrinidae } \\
\end{array}$ & \\
\hline Gyrinus (Gyrinus) caspıus Ménétries, 1832 & GY.MI \\
\hline Gyrinus (Gyrinus) dejeani Brullé, 1832 & GY.DE \\
\hline Gyrinus (Gyrinus) distinctus Aubé, 1836 & GY.DI \\
\hline Gyrinus (Gyrinus) urinator Iliiger, 1807 & GY.UR \\
\hline Aulonogyrus striatus (Fahricius, 1792) & AU.ST \\
\hline Orectochilus villosus (Müller, 1776) & OR.VI \\
\hline F. Haliplidae & \\
\hline Peltodytes rotundatus (Aube, 1836 ) & PE.RO \\
\hline Haliplus (Haliplidus) obliquus (Fabricius, 1787) & НАОВ \\
\hline Haliplus (Neohaliplus) lineatocollis (Marsham, 1802) & HALI \\
\hline Haliplus (Liaphlus) mucronatus Stephens, 1832 & HAMU \\
\hline F. Noteridae & \\
\hline Noterus laevis Sturm, 1834 & NO.LA \\
\hline F. Dytiscidae & \\
\hline Hyphydrus aubei Ganglbauer, 1892 & HY.A' \\
\hline Hydrovatus cuspidatus (Kunze, 1818) & HY.CU \\
\hline Yola (Yola) bicarinata (Latreille, 1804) & Yo.BI \\
\hline Bidessus minutissimus (Germar, 1824) & BI.MI \\
\hline Bidessus pumilus* (Ảubé, 1836) & \\
\hline Hydroglyphus pusilllus (Fabricius, 1781) & HY.PU \\
\hline Hydroglyphus signatellus (Klug, 1834) & HY.SI \\
\hline Hygrotus (Coelambus) confluens (Fabricius, 1787) & HY.CO \\
\hline Hygrotus (Coelamhus) impressopunctatus (Schaller, 1783) & HY.IM \\
\hline Herophydrus musicus (Klug, 1833) & HE.MU \\
\hline Hydropoms discretus Fairmaire, 1859 & HY.DI \\
\hline Hydropoms limbatus Aubé, 1836 & HY.LI \\
\hline Hydropoms lucasi Reiche, 1866 & HY.LU \\
\hline Hydroporus marginatus (Duftschmid, 1805) & HY.MA \\
\hline Hydroporus nigrita (Fabricius, 1792) & HY.NI \\
\hline Hydropoms tessellatus Drapiez, 1819 & HY.TE \\
\hline Graptodytes fractus (Sharp, 1880-82) & GR.FR \\
\hline Graptodytes varuus (Auhé, 1836) & GR.VA \\
\hline Stictonectes epipleuricus (Seidlitz, 1887) & ST.EP \\
\hline Stictonectes lepidus (Olivier, 1795) & ST.LE \\
\hline Stictonectes optatus (Seidlitz, 1887) & ST.OP \\
\hline Deroncetes depressicollis (Rosenhauer, 1856) & DE.DE \\
\hline Deronectes fairmairei (Leprieur, 1876) & DE.FA \\
\hline Deronectes hispanicus (Rosenhauer, 1856) & DE.HI \\
\hline Deronectes moestus (Fairmaire, 1858) & DE.MO \\
\hline Stictotarsus of griseostriatus (De Geer, 1774) & ST.GR \\
\hline Nebriopoms baeticus (Schaum,, 1864) & NE.BA \\
\hline Nebrioponis cazorlensis lagar, Fresneda \& Hernando 1987, & NE.CA \\
\hline Nebrioporus cercsyi (Aubé, 1836) & NE.CE \\
\hline Nebriuporus ciarki (Wollaston, 1862) & $\mathrm{NECJ}$ \\
\hline Oreodytes davisii (Curtis, 1831) & OR.DA \\
\hline Oreodytes sanmarkii (Sahlberg, 1826) & OR.SA \\
\hline Agabus brunneus (Fabricius, 1798) & AG.BR \\
\hline Agabus br. rufulus** sensu Millán, 1991 & \\
\hline Agabus didymus (Olivier, 1795) & AG.DI \\
\hline Agabus biguttatus (Olivier, 1795) & AG.BG \\
\hline Agabus guttatus (Paykull, 1798) & AG GU \\
\hline Agabus nitidus (Fabricius. 1801) & AG.NI \\
\hline Agabus bipustulatus (Linnaeus, 1767) & AG.BP \\
\hline Agabus chalconatiis (Panzer, 1796) & $\mathrm{AGCH}$ \\
\hline Agabus montanus (Stephens, 1928) & AG.MO \\
\hline Agabus nebulosus (Forster, 1771) & AG NE \\
\hline Agabus paludosus (Fabricius, 1801) & AG.PA \\
\hline Ilybius meridionalis Aubé. 1836 & IL.ME \\
\hline Rhantus suturalis (McLeay, 1825) & RH SU \\
\hline Colymbetes fuscus' (Linnaeus, 1758) & \\
\hline Meladema coriacea Castelnau, 1834 & ME.CO \\
\hline Laccophilus hyalinus (De Geer, 1774) & LAHY \\
\hline Laccophilus iiiinutus (Linnaeus, 1758) & LAMI \\
\hline Laccophilus ponticus Sharp, 1882 & LA.PO \\
\hline Eretes sticticus (Linnaeus, 1767) & ER.ST \\
\hline liydaticus leander (Rossi, 1790) & HY LE \\
\hline Dytisus circumtlexus Fabricius. 1801 & DY.Cl \\
\hline D)tiscus pisanus Castelnau, 1834 & DY PI \\
\hline Cybister lateralimarginalis (De Geer, 1774) & CY LA \\
\hline Crbister tripunctatus africanus Castelnau, 1834 & CY.TR \\
\hline
\end{tabular}


Apéndice 2. Tabla de contingencia de la frecuencia de especies para cada rango de las variables ambientales. RAN: rango de las variables ambientales. spp: frecuencia de especies para cada rango. APAK: frecuencia de especies en la zona de estudio.

Appendix 2. Contingence table of the species frequency for each range of the environmental variables. RAN: range of the environmental variables. spp: species number in each range. APAK. Species frequency in the stody area

\begin{tabular}{|c|c|c|c|c|c|c|c|c|c|c|c|}
\hline \multirow[b]{2}{*}{ RAN : } & \multicolumn{2}{|c|}{ ALTITUD } & \multirow[b]{2}{*}{2} & \multirow[b]{2}{*}{3} & \multicolumn{3}{|c|}{ PERSIST. AGUA } & \multicolumn{3}{|c|}{ PROFUNDIDAD } & \multirow[b]{2}{*}{ APAR. } \\
\hline & 0 & 1 & & & 0 & 1 & 2 & $\mathrm{O}$ & 1 & 2 & \\
\hline PERO & 0.00 & 33.48 & 82.82 & 0.00 & 0.00 & 0.00 & 56.18 & 0.00 & 53.30 & 42.02 & 7 \\
\hline HAOB & 0.00 & 0.00 & 144.93 & 0.00 & 0.00 & 0.00 & 56.18 & 0.00 & 74.63 & 0.00 & 1 \\
\hline HALI & 16.49 & 27.75 & 55.53 & 68.72 & 15.58 & 42.06 & 44.10 & 42.67 & 43.24 & 32.99 & 107 \\
\hline HAMU & 0.00 & 28.70 & 68.03 & 48.02 & 6.80 & 40.82 & 45.86 & 35.49 & 51.78 & 21.01 & 49 \\
\hline AUST & 0.00 & 44.34 & 47.00 & 31.80 & 27.03 & 47.30 & 41.00 & 41.13 & 46.39 & 27.82 & 37 \\
\hline GYCA & 0.00 & 0.00 & 144.93 & 0.00 & 0.00 & 0.00 & 56.18 & 0.00 & 74.63 & 0.00 & 3 \\
\hline GYDE & 0.00 & 6.51 & 72.46 & 122.55 & 27.78 & 20.83 & 46.82 & 36.23 & 55.97 & 12.25 & 12 \\
\hline GYDI & 0.00 & 0.00 & 48.311 & 196.08 & 55.56 & 0.00 & 46.82 & 36.23 & 37.31 & 49.02 & 6 \\
\hline GYUR & 0.00 & 36.76 & 59.68 & 34.60 & 0.00 & 44.12 & 46.27 & 63.94 & 52.68 & 0.00 & 17 \\
\hline ORVI & 0.00 & 8.37 & 82.82 & 94.54 & 11.90 & 17.86 & 50.16 & 31.06 & 53.30 & 21.01 & 28 \\
\hline NOLA & 45.25 & 54.09 & 22.30 & 22.62 & 0.00 & 19.23 & 51.86 & 33.44 & 34.44 & 56.56 & 13 \\
\hline HYAU & 117.65 & 62.50 & 0.00 & 0.00 & 133.33 & 0.00 & 33.71 & 0.00 & 44.78 & 58.82 & 5 \\
\hline HYCU & 0.00 & 78.13 & 0.00 & 0.00 & 0.00 & 0.00 & 56.18 & 0.00 & 74.63 & 0.00 & 1 \\
\hline YOBI & 17.83 & 42.61 & 43.92 & 35.65 & 60.61 & 45.45 & 35.75 & 32.94 & 47.49 & 31.19 & 33 \\
\hline BIMI & 0.00 & 28.04 & 74.32 & 45.25 & 17.09 & 44.87 & 44.66 & 39.02 & 47.84 & 30.17 & 39 \\
\hline HYPU & 43.57 & 56.42 & 26.84 & 5.45 & 43.21 & 46.30 & 38.49 & 32.21 & 41.46 & 43.57 & 54 \\
\hline HYSI & 117.65 & 54.69 & 14.49 & 0.00 & 0.00 & 100.00 & 33.71 & 21.74 & 37.31 & 58.82 & 10 \\
\hline HYCo & 0.00 & 69.44 & 16.10 & 0.00 & 85.19 & 27.78 & 18.73 & 24.15 & 24.88 & 81.70 & 9 \\
\hline HYIM & 0.00 & 0.00 & 144.93 & 0.00 & 0.00 & 0.00 & 56.18 & 0.00 & 74.63 & 0.00 & 1 \\
\hline HEMU & 147.06 & 52.08 & 6.04 & 12.25 & 111.11 & 10.42 & 35.11 & 9.06 & 37.31 & 67.40 & 24 \\
\hline HYDI & 0.00 & 3.91 & 43.48 & 205.88 & 16.67 & 37.50 & 47.75 & 65.22 & 37.31 & 36.76 & 20 \\
\hline HYLI & 196.08 & 52.08 & 0.00 & 0.00 & 111.11 & 83.33 & 18.73 & 0.00 & 74.63 & 0.00 & 3 \\
\hline HYLU & 0.00 & 0.00 & 43.48 & 235.29 & 0.00 & 50.00 & 50.56 & 21.74 & 52.24 & 44.12 & 10 \\
\hline HYMA & 0.00 & 15.63 & 86.96 & 58.82 & 0.00 & 0.00 & 56.18 & 43.48 & 59.70 & 0.00 & 5 \\
\hline HYNI & 0.00 & 0.00 & 0.00 & 294.12 & 0.00 & 0.00 & 56.18 & 43.48 & 44.78 & 29.41 & 5 \\
\hline HYTE & 0.00 & 0.00 & 0.00 & 294.12 & 0.00 & 0.00 & 56.18 & 0.00 & 37.31 & 73.53 & 2 \\
\hline GRPR & 0.00 & 13.02 & 72.46 & 98.04 & 0.00 & 125.00 & 28.09 & 144.93 & 12.44 & 24.51 & 6 \\
\hline GRVA & 0.00 & 0.00 & 79.05 & 133.69 & 0.00 & 0.00 & 56.18 & 19.76 & 54.27 & 26.74 & II \\
\hline STEP & 0.00 & 0.00 & 28.99 & 235.29 & 33.33 & 0.00 & 50.56 & 43.48 & 37.31 & 44.12 & 10 \\
\hline STLE & 0.00 & 0.00 & 0.00 & 294.12 & 0.00 & 0.00 & 56.18 & 0.00 & 0.00 & 147.06 & 1 \\
\hline STOP & 0.00 & 36.06 & 33.44 & 90.50 & 0.00 & 57.69 & 43.22 & 50.17 & 40.18 & 33.94 & 13 \\
\hline DEDE & 0.00 & 0.00 & 0.00 & 294.12 & 0.00 & 0.00 & 56.18 & 0.00 & 74.63 & 0.00 & 1 \\
\hline gpp. & 17 & 40 & 44 & 46 & 28 & 36 & 63 & 42 & 56 & & \\
\hline Est. & 17 & 128 & 69 & 34 & 30 & 40 & 178 & 46 & 134 & 8 & \\
\hline & AI & ITUD & & & & SIST. & AGUA & & FUNDI & & \\
\hline RAN: & 0 & 1 & 2 & 3 & 0 & 1 & 2 & 0 & 1 & 2 & \\
\hline DEPA & 0.00 & 65.10 & 24.15 & 0.00 & 0.00 & 125.00 & $28.09^{\prime}$ & 36.23 & 62.19 & 0.00 & 6 \\
\hline EHI & 98.04 & 0.00 & 48.31 & 147.06 & 0.00 & 0.00 & 56.18 & 36.23 & 37.31 & 49.02 & 6 \\
\hline DEMO & 0.00 & 0.00 & 64.41 & 163.40 & 0.00 & 27.78 & 49.94 & 24.15 & 41.46 & 49.02 & 9 \\
\hline NECA & 0.00 & 0.00 & 72.46 & 147.06 & 0.00 & 31.25 & 49.16 & 13.59 & 60.63 & 18.38 & 16 \\
\hline NECL & 0.00 & 34.91 & 58.59 & 43.80 & 14.18 & 47.87 & 43.03 & 41.63 & 50.81 & 28.77 & 47 \\
\hline TGR & 0.00 & 78.13 & 0.00 & 0.00 & 0.00 & 0.00 & 56.18 & 0.00 & 0.00 & 147.06 & 1 \\
\hline NEBA & 0.00 & 74.73 & 6.30 & 0.00 & 57.97 & 86.96 & 26.87 & 66.16 & 35.69 & 31.97 & 23 \\
\hline ECE & 504.20 & 11.16 & 0.00 & 0.00 & 142.86 & 0.00 & 32.10 & 0.00 & 74.63 & 0.00 & 7 \\
\hline RDA & 0.00 & 0.00 & 0.00 & 294.12 & 0.00 & 0.00 & 56.18 & 0.00 & 37.31 & 73.53 & 2 \\
\hline ORSE & 0.00 & 0.00 & 0.30 & 294.12 & 0.00 & 0.00 & 56.18 & 0.00 & 0.00 & 147.06 & 1 \\
\hline LAHY & 39.22 & 46.22 & 39.86 & 19.61 & 30.56 & 35.42 & 43.07 & 28.99 & 43.53 & 41.67 & 120 \\
\hline AMI & 45.25 & 60.10 & 22.30 & 0.00 & 76.92 & 38.46 & 34.57 & 16.72 & 45.92 & 45.25 & 73 \\
\hline LAPO & 588.24 & 0.00 & 0.00 & 0.00 & 0.00 & 0.00 & 56.18 & 0.00 & 0.00 & 147.06 & 1 \\
\hline AGBR & 0.00 & 42.07 & 33.44 & 67.87 & 25.64 & 76.92 & 34.57 & 83.61 & 40.18 & 11.31 & 13 \\
\hline GDI & 0.00 & 24.04 & 78.04 & 45.25 & 0.00 & 38.46 & 47.54 & 16.72 & 63.15 & 11.31 & 13 \\
\hline GBG & 0.00 & 11.16 & 62.11 & 126.05 & 0.00 & 35.71 & 48.15 & 62.11 & 42.64 & 21.01 & 7 \\
\hline GGU & 0.00 & 0.00 & 0.00 & 294.12 & 0.00 & 0.00 & 56.18 & 217.39 & 0.00 & 0.00 & 1 \\
\hline GNI & 0.00 & 0.00 & 0.00 & 294.12 & 0.00 & 0.00 & 56.18 & 0.00 & 59.70 & 29.41 & 5 \\
\hline GBP & 0.00 & 17.36 & 64.41 & 98.04 & 37.04 & 55.56 & 37.45 & 48.31 & 41.46 & 32.68 & 9 \\
\hline $\mathrm{GCH}$ & 0.00 & 0.00 & 0.00 & 294.12 & 0.00 & 250.00 & 0.0 & 0.00 & 74.63 & 0.00 & 1 \\
\hline BMO & 0.00 & 0.00 & 0.00 & 294.12 & 0.00 & 0.00 & & 0.00 & 0.00 & 147.06 & 1 \\
\hline GNE & 0.00 & 39.06 & 36.23 & 73.53 & 0.00 & 62.50 & & 0.00 & 55.97 & 36.76 & 4 \\
\hline GPA & 0.00 & 0.00 & 48.311 & 96.08 & 0.00 & 0.00 & 56.18 & 0.00 & 49.75 & 49.02 & 3 \\
\hline ILME & 0.00 & 0.00 & 28.99 & 235.29 & 66.67 & 0.00 & 44.94 & 43.48 & 29.85 & 58.82 & 5 \\
\hline isu & 42.02 & 55.80 & 31.06 & 0.00 & 95.24 & 35.71 & 32.10 & 46.58 & 37.31 & 42.02 & 14 \\
\hline $\mathrm{CO}$ & 61.92 & 41.12 & 45.77 & 15.48 & 70.18 & 13.16 & 41.40 & 45.77 & 47.13 & 23.22 & 19 \\
\hline RST & 117.65 & 54.69 & 0.00 & 29.41 & 33.33 & 75.00 & 33.71 & 43.48 & 52.24 & 14.71 & 10 \\
\hline YLE & 39.22 & 52.08 & 19.32 & 39.22 & 111.11 & 83.33 & 18.73 & 57.97 & 39.80 & 29.41 & 15 \\
\hline DYCI & 0.00 & 39.06 & 0.00 & 147.06 & 166.67 & 0.00 & 28.09 & 0.00 & 37.31 & 73.53 & \\
\hline YPI & 0.00 & & 108.10 & & 0.00 & 62.50 & & 108.70 & 37.31 & 0.00 & 4 \\
\hline CYTR & 0.00 & 78.13 & 0.00 & 0.00 & 0.00 & 0.00 & 56.18 & 0.00 & 0.00 & 147.05 & 1 \\
\hline CYLA & 0.00 & 78.13 & 0.00 & 0.00 & 0.00 & 0.00 & 56.18 & 0.00 & 0.00 & 147.06 & 1 \\
\hline & 17 & 40 & 4 & 46 & 28 & 36 & & 42 & 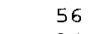 & 51 & \\
\hline & 17 & 128 & 69 & 34 & 30 & 40 & 178 & 46 & 134 & 68 & \\
\hline
\end{tabular}




\begin{tabular}{|c|c|c|c|c|c|c|c|c|c|c|c|c|c|c|}
\hline \multirow[b]{2}{*}{ RAN: } & \multicolumn{2}{|c|}{ VELOCIDAD } & \multicolumn{2}{|c|}{ D CORRIENTE } & \multicolumn{3}{|c|}{ TIPO SUSTRATO } & \multirow[b]{2}{*}{3} & \multirow[b]{2}{*}{4} & \multicolumn{4}{|c|}{ SEDIMENTO OKGANICO } & \multirow[b]{2}{*}{ APAR } \\
\hline & 0 & 1 & 2 & 3 & 0 & 1 & 2 & & & 0 & 1 & 2 & 3 & \\
\hline PERO & 20.70 & 38.61 & 70.55 & 0.00 & 45.11 & 39.68 & 0.00 & 91.19 & 0.00 & 60.15 & 71.43 & 98.52 & 0.00 & 7 \\
\hline HAOB & 0.00 & 0.00 & 123.46 & 0.00 & 0.00 & 0.00 & 0.00 & 212.77 & 0.00 & 0.00 & 0.30 & 344.83 & 0.00 & 1 \\
\hline HALI & 20.32 & 35.36 & 56.54 & 58.41 & 25.58 & 33.75 & 56.43 & 61.64 & 27.49 & 53.12 & 42.06 & 54.79 & 24.26 & 107 \\
\hline HAMU & 17.75 & 38.61 & 55.43 & 59.52 & 23.63 & 34.01 & 30.80 & 82.50 & 60.02 & 60.15 & 51.02 & 49.26 & 17.66 & 49 \\
\hline AUST & 19.58 & 29.22 & 63.40 & 56.31 & 19.91 & 60.06 & 56.09 & 63.25 & 0.00 & 65.43 & 81.08 & 9.32 & 18.19 & 37 \\
\hline GYCA & 0.00 & 45.05 & 82.30 & 0.00 & 0.00 & 92.59 & 0.00 & 141.84 & 0.00 & 70.18 & 166.67 & 0.00 & 0.00 & 3 \\
\hline GYDE & 0.00 & 22.52 & 72.02 & 104.17 & 0.00 & 23.15 & 62.89 & 124.11 & 0.00 & 70.18 & 125.00 & 0.00 & 8.01 & 12 \\
\hline GYDI & 24.15 & 0.00 & 102.88 & 0.00 & 0.00 & 46.30 & 62.89 & 106.38 & 0.00 & 87.72 & 83.33 & 0.00 & 0.00 & 6 \\
\hline GYUR & 8.53 & 23.85 & 79.88 & 49.02 & 24.77 & 32.68 & 55.49 & 62.58 & 34.60 & 74.30 & 0.00 & 60.85 & 11.31 & 17 \\
\hline ORVI & 5.18 & 4.83 & 92.59 & 74.40 & 0.00 & 0.00 & 94.34 & 106.38 & 0.00 & 63.91 & 125.00 & 36.95 & 3.43 & 28 \\
\hline NOLA & 33.44 & 62.37 & 28.49 & 32.05 & 56.68 & 21.37 & 29.03 & 32.73 & 45.25 & 8.10 & 76.92 & 79.58 & 51.78 & 13 \\
\hline HYAU & 115.94 & 27.03 & 0.00 & 0.00 & 105.26 & 0.00 & 0.00 & 0.00 & 0.00 & 0.00 & 0.00 & 68.97 & 76.92 & 5 \\
\hline HYCU & 0.00 & 135.14 & 0.00 & 0.00 & 0.00 & 0.00 & 0.00 & 212.77 & 0.00 & 0.00 & 0.00 & 344.83 & 0.00 & 1 \\
\hline YOBI & 39.53 & 28.67 & 59.86 & 12.63 & 41.47 & 16.84 & 40.02 & 64.47 & 17.83 & 35.09 & 60.61 & 31.35 & 43.71 & 33 \\
\hline BIMI & 22.30 & 34.65 & 69.64 & 21.37 & 26.99 & 21.37 & 62.89 & 65.47 & 30.17 & 70.18 & 64.10 & 17.68 & 17.26 & 39 \\
\hline HYPU & 45.63 & 37.54 & 43.44 & 23.15 & 46.78 & 36.01 & 45.42 & 31.52 & 21.79 & 33.14 & 46.30 & 44.70 & 44.52 & 54 \\
\hline HYSI & 72.46 & 54.05 & 12.35 & 0.00 & 63.16 & 55.56 & 37.74 & 0.00 & 0.00 & 10.53 & 0.00 & 0.00 & 86.54 & 10 \\
\hline HYCO & 112.72 & 30.03 & 0.00 & 0.00 & 93.57 & 0.00 & 20.96 & 0.00 & 0.00 & 11.70 & 0.00 & 38.31 & 74.79 & 9 \\
\hline HYIM & 144.93 & 0.00 & 0.00 & 0.00 & 105.26 & 0.00 & 0.00 & 0.00 & 0.00 & 0.00 & 0.00 & 0.00 & 96.15 & 1 \\
\hline HEMU & 96.62 & 16.89 & 25.72 & 0.00 & 87.72 & 23.15 & 15.72 & 0.00 & 0.00 & 17.54 & 0.00 & 57.47 & 64.10 & 24 \\
\hline BYDI & 21.74 & 27.03 & 61.73 & 83.33 & 21.05 & 13.89 & 66.04 & 74.47 & 58.82 & 73.68 & 100.00 & 17.24 & 9.62 & 20 \\
\hline HYLI & 96.62 & 0.00 & 41.15 & 0.00 & 35.09 & 92.59 & 0.00 & 70.92 & 0.00 & 0.00 & 0.00 & 0.00 & 96.15 & 3 \\
\hline HYLU & 43.48 & 27.03 & 49.39 & 83.33 & 10.53 & 27.78 & 56.60 & 106.38 & 58.82 & 73.68 & 150.00 & 0.00 & 9.62 & 10 \\
\hline HYMA & 0.00 & 54.05 & 49.38 & 83.33 & 0.00 & 111.11 & 0.00 & 127.66 & 0.00 & 21.05 & 100.00 & 68.97 & 38.46 & 5 \\
\hline HYNI & 0.00 & 0.00 & 98.77 & 83.33 & 0.00 & 0.00 & 75.47 & 127.66 & 0.00 & 84.21 & 100.00 & 0.00 & 0.00 & 5 \\
\hline HYTE & 0.00 & 0.00 & 123.46 & 0.00 & 0.00 & 0.00 & 188.68 & 0.00 & 0.00 & 52.63 & 250.00 & 0.00 & 0.00 & 2 \\
\hline GRFR & 24.15 & 90.09 & 20.58 & 0.00 & 17.54 & 92.59 & 52.89 & 0.00 & 98.04 & 87.72 & 0.00 & 57.47 & 0.00 & 6 \\
\hline GRVA & 13.18 & 0.00 & 101.01 & 37.88 & 0.00 & 25.25 & 51.45 & 135.40 & 0.00 & 95.69 & 0.00 & 31.35 & 0.00 & 11 \\
\hline STEP & 0.00 & 27.03 & 74.07 & 83.33 & 0.00 & 0.00 & 56.60 & 148.94 & 0.00 & 84.21 & 50.00 & 34.48 & 0.00 & 10 \\
\hline STLE & 144.93 & 0.00 & 0.00 & 0.00 & 0.00 & 277.78 & 0.00 & 0.00 & 0.00 & 0.00 & 500.00 & 0.00 & 0.00 & 1 \\
\hline STOP & 33.44 & 31.19 & 66.48 & 0.00 & 24.29 & 64.10 & 29.03 & 49.10 & 90.50 & 72.87 & 38.46 & 26.53 & 14.79 & 13 \\
\hline DEDE & 0.00 & 0.00 & 123.46 & 0.00 & 0.00 & 0.00 & 0.00 & 212.77 & 0.00 & 105.26 & 0.00 & 0.00 & 0.00 & 1 \\
\hline ApP. & 43 & 44 & 50 & 29 & 38 & 37 & 42 & 40 & 23 & 49 & 35 & 39 & 40 & \\
\hline Est. & 69 & 74 & 81 & 24 & 95 & 36 & 53 & 47 & 17 & 95 & 20 & 29 & 104 & \\
\hline
\end{tabular}

\begin{tabular}{|c|c|c|c|c|c|c|c|c|c|c|c|c|c|c|}
\hline \multirow[b]{2}{*}{ RAN: } & \multicolumn{4}{|c|}{ VELOCIDAD CORRIENTE } & \multicolumn{5}{|c|}{ TIPO SUSTRATO } & \multicolumn{4}{|c|}{ SEDIMENTO ORGANICO } & \multirow[b]{2}{*}{ APAR. } \\
\hline & 0 & 1 & 2 & 3 & 0 & 1 & 2 & 3 & 4 & 0 & 1 & 2 & 3 & \\
\hline DEFA & 24.15 & 45.05 & 61.73 & 0.00 & 70.18 & 46.30 & 31.45 & 0.00 & 0.00 & 52.63 & 0.00 & 57.47 & 32.05 & 6 \\
\hline DEMO & 0.00 & 15.02 & 68.59 & 138.89 & 0.00 & 0.00 & 83.86 & 94.56 & 65.36 & 93.57 & 55.56 & 0.00 & 0.00 & 9 \\
\hline $\mathrm{NECA}$ & 0.00 & 0.00 & 84.88 & 130.21 & 0.00 & 0.00 & 35.38 & 172.87 & 0.00 & 65.79 & 156.25 & 21.55 & 0.00 & 16 \\
\hline NECL & 21.58 & 14.38 & 84.06 & 26.60 & 26.88 & 35.46 & 56.20 & 63.38 & 12.52 & 58.23 & 53.19 & 66.03 & 14.32 & 47 \\
\hline STGR & 144.93 & 0.00 & 0.00 & 0.00 & 105.26 & 0.00 & 0.00 & 0.00 & 0.00 & 0.00 & 0.00 & 344.83 & 0.00 & 1 \\
\hline ORDA & 0.00 & 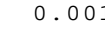 & 123.46 & 0.00 & 0.00 & 0.00 & 94.34 & 106.38 & 0.00 & 52.63 & 250.00 & & 0.00 & 2 \\
\hline ORSE & 0.00 & 0.00 & 123.46 & 0.00 & 0.00 & 0.00 & 188.68 & 0.00 & 0.00 & 0.00 & 500.00 & 0.00 & 0.00 & 1 \\
\hline LAEY & 30.19 & 46.17 & $50.4:$ & 17.36 & 45.61 & 34.72 & 34.59 & 40.78 & 39.22 & 32.46 & 37.50 & 57.47 & 43.27 & 120 \\
\hline LAMI & 66.89 & 31.19 & 37.99 & 0.00 & 80.97 & 42.74 & 14.51 & & 0.00 & 24.29 & & 26.53 & 66.57 & 13 \\
\hline LAPO & 0.00 & 135.14 & 00 & 0.00 & 105.26 & 0.00 & 0.00 & 0.00 & 0.00 & 0.00 & 0.00 & 0.00 & 96.15 & 1 \\
\hline AGBR & 11.15 & 62.37 & 47.46 & 32.05 & 40.49 & 42.74 & 43.54 & 16.37 & 90.50 & 64.78 & 0.00 & 26.53 & 29.59 & 13 \\
\hline AGDI & 0.00 & 20.79 & 85.47 & 64.10 & 16.19 & 21.37 & 72.57 & 81.83 & 0.00 & 56.68 & 115.38 & 26.53 & 14.79 & 13 \\
\hline AGMO & 144.93 & 0.00 & .00 & 0.00 & 0.00 & 277.78 & 0.00 & 0.00 & 0.00 & 0.00 & 500.00 & 0.00 & 0.00 & 1 \\
\hline AGNE & 08.70 & 33.78 & .00 & 0.00 & 78.95 & 0.00 & 0.00 & 0.00 & 147.06 & 0.00 & 125.00 & 86.21 & 48.08 & 4 \\
\hline & 0.00 & 45.05 & 0.00 & 277.78 & 0.00 & 0.00 & 0.00 & 141.84 & 196.08 & 35.09 & 333.33 & 0.00 & 0.00 & 3 \\
\hline ILME & 0.00 & .00 & 74.07 & 166.67 & 0.00 & 0.00 & 75.47 & 127.66 & 0.00 & 63.16 & 100.00 & & & 5 \\
\hline & 72.46 & 28.96 & 5.27 & .00 & 75.19 & 19.84 & 40.43 & 0.00 & 0.00 & 22.56 & 0.00 & 73.89 & 54.95 & 14 \\
\hline MECO & 30.51 & 49.79 & 1.98 & 0.00 & 49.86 & 29.24 & & 33.59 & 61.92 & 60.94 & & & & 19 \\
\hline ERST & 86.96 & & & 0.00 & 84.21 & 27.78 & 18.87 & 0.00 & 0.00 & 21.05 & 0.00 & 34.48 & 67.31 & 10 \\
\hline HYLE & 38.65 & 63.06 & 24.69 & 27.78 & 70.18 & 37.04 & 25.16 & 14.18 & 0.00 & 42.11 & 0.00 & 45.98 & 44.87 & 15 \\
\hline DYCI & 144.93 & 0.00 & 0.00 & 0.00 & 105.26 & 0.00 & 0.00 & 0.00 & 0.00 & 0.00 & 0.00 & 0.00 & 96.15 & 2 \\
\hline DYPI & 36.23 & 33.78 & 61.73 & 0.00 & 26.32 & 0.001 & 141.51 & 0.00 & 0.00 & 78.95 & 0.00 & 0.00 & 24.04 & 4 \\
\hline CYTR & 144.93 & 0.00 & 0.00 & 0.00 & 0.00 & 0.00 & 0.00 & 0.00 & 588.24 & 0.00 & 0.00 & 0.00 & 96.15 & 1 \\
\hline CYLA & 44.93 & 0.00 & 0.00 & 0.00 & 0.00 & 277.78 & 0.00 & 0.00 & 0.00 & 0.00 & 0.00 & 344.83 & 0.00 & 1 \\
\hline & 43 & 44 & 5 & 29 & & & & & & & & & & \\
\hline & 69 & 74 & 81 & 24 & 95 & 36 & 53 & 47 & 17 & 95 & 20 & 29 & 104 & \\
\hline
\end{tabular}




\begin{tabular}{|c|c|c|c|c|c|c|c|c|c|c|c|c|c|c|c|}
\hline \multirow[b]{2}{*}{ RAN : } & \multicolumn{3}{|c|}{ TIPO MACROFITOS } & \multirow[b]{2}{*}{3} & \multirow[b]{2}{*}{4} & \multicolumn{4}{|c|}{ COBERTURA MACROFITOS } & \multirow[b]{2}{*}{4} & \multicolumn{4}{|c|}{ VEGETACION RIBERA } & \multirow[b]{2}{*}{ MPAR } \\
\hline & 0 & 1 & 2 & & & 0 & 1 & 2 & 3 & & 0 & 1 & 2 & 3 & \\
\hline PERO & 28.57 & 0.00 & 20.701 & 109.89 & 36.63 & 28.01 & 19.31 & 54.95 & 64.94 & 52.91 & 0.30 & 0.30 & 64.35 & 42.64 & 7 \\
\hline HAOB & 0.00 & 0.00 & 0.001 & 192.31 & 0.00 & 0.00 & 0.00 & 0.00 & 227.27 & 0.00 & 0.00 & 0.00 & 0.00 & 149.25 & 1 \\
\hline HALI & 14.95 & 59.03 & 43.34 & 44.93 & 43.13 & 14.66 & 44.20 & 53.92 & 46.73 & 41.54 & 11.21 & 22.85 & 37.05 & 68.35 & 107 \\
\hline HAMU & 4.08 & 48.34 & 32.53 & 82.42 & 36.63 & 4.00 & 35.85 & 43.17 & $69 \quad 57$ & 68.03 & 0.00 & $13.6,1$ & 42.29 & 70.06 & 49 \\
\hline AUST & 43.24 & 56.90 & 23.50 & 31.19 & 62.37 & 42.40 & 40.18 & 46.78 & 12.29 & 70.07 & 10.81 & 18.02 & 53.4 & $14.2 \%$ & $3 \%$ \\
\hline GYCA & 0.00 & 175.44 & 48.31 & 0.00 & 0.00 & 0.00 & 90.09 & 64.10 & 0.00 & 0.00 & 0.00 & i] 00 & 30.03 & 94.50 & i \\
\hline GYDE & 16.67 & 109.65 & 36.23 & 16.03 & 42.74 & 16.34 & 67.57 & 48.08 & 37.88 & 0.00 & 0.00 & 0.00 & 7.51 & 136.82 & 12 \\
\hline GYDI & 33.33 & 131.58 & 24.15 & 0.00 & 42.74 & 32.68 & 67.57 & 32.05 & 37.88 & 0.00 & 0.00 & 0.00 & 15.02 & 124.38 & 6 \\
\hline GYUR & 11.76 & 61.92 & 59.68 & 56.56 & 0.00 & 11.53 & 31.80 & 56.56 & 66.84 & 43.57 & 0.00 & 26.14 & 42.40 & 61.46 & 17 \\
\hline ORVI & 0.00 & 93.98 & 31.06 & 41.21 & 54.95 & 0.00 & 33.78 & 75.55 & 56.82 & 39.68 & 0.00 & 0.00 & 16.09 & 122.60 & 28 \\
\hline NOLA & 30.77 & 40.49 & 66.89 & 14.79 & 39.45 & 30.17 & 31.19 & 59.17 & 34.97 & 56.98 & 30.771 & 02.56 & 27.72 & 22.96 & 13 \\
\hline HYAU & 0.00 & 0.00 & 86.96 & 0.001 & 102.56 & 0.00 & 54.05 & 76.92 & 45.45 & 0.00 & 80.00 & 44.44 & 54.05 & 0.00 & 5 \\
\hline $\mathrm{HYCU}$ & 0.00 & 0.00 & 144.93 & 0.00 & 0.00 & 0.00 & 0.00 & 0.00 & 227.27 & 0.00 & 0.00 & 222.22 & 0.00 & 0.00 & 1 \\
\hline YOBI & 12.12 & 31.90 & 39.53 & 87.41 & 23.31 & 11.88 & 40.95 & 46.62 & 55.10 & 56.12 & 24.24 & 26.94 & 40.95 & 54.27 & 33 \\
\hline BIMI & 20.51 & 40.49 & 33.41 & 73.96 & 39.45 & 20.11 & 62.37 & 44.38 & 40.79 & 18.99 & 20.51 & 5.70 & 43.89 & 68.89 & 39 \\
\hline HYPU & 48.15 & 14.62 & 48.31 & 28.49 & 56.98 & 50.84 & 52.55 & 24.93 & 16.84 & 54.87 & 37.04 & 37.04 & 53.39 & 22.11 & 54 \\
\hline HYS I & 20.00 & 0.00 & 72.46 & 19.23 & 76.92 & 19.61 & 54.05 & 19.23 & 90.91 & 0.00 & 80.00 & 66.67 & 45.05 & 0.00 & 10 \\
\hline $\mathrm{HYCO}$ & 88.89 & 29.24 & 16.10 & 42.74 & 28.49 & 87.15 & 30.03 & 42.74 & 25.25 & 0.00 & 88.89 & 0.00 & 70.07 & 0.00 & 9 \\
\hline HYIM & 0.00 & 0.00 & 144.93 & 0.00 & 0.00 & 0.00 & 135.14 & 0.00 & 0.00 & 0.00 & 0.00 & 222.22 & 0.00 & 0.00 & 1 \\
\hline FEMU & 50.00 & 21.93 & 42.27 & 16.03 & 74.79 & 49.02 & 28.15 & 24.04 & 47.35 & 77.16 & 50.00 & 83.33 & 41.29 & 6.22 & 24 \\
\hline HYDI & 10.00 & 118.42 & 28.99 & 57.69 & 12.82 & 9.80 & 40.54 & 38.46 & 68.18 & 74.07 & 20.00 & 0.00 & 18.02 & 119.40 & 20 \\
\hline HYLI & 66.67 & 0.00 & 48.31 & 0.00 & 85.47 & 65.36 & 45.05 & 64.10 & 0.00 & 0.00 & 133.33 & 0.00 & 60.06 & 0.00 & 3 \\
\hline HYLU & 0.00 & 131.58 & 43.48 & 57.69 & 0.00 & 0.00 & 40.54 & 19.23 & 68.18 & 148.15 & 0.00 & 0.00 & 27.03 & 119.40 & 10 \\
\hline HYMA & 0.00 & 52.63 & 0.00 & 38.46 & 153.85 & 0.00 & 27.031 & 15.38 & 0.00 & 74.07 & 80.00 & 0.00 & 18.02 & 89.55 & 5 \\
\hline EYNI & 0.00 & 105.26 & 57.97 & 0.00 & 51.28 & 0.00 & 54.05 & 38.46 & 45.45 & 74.07 & 0.30 & 0.00 & 0.00 & 149.25 & 5 \\
\hline BYTE & 0.001 & 131.58 & 72.46 & 0.00 & 0.00 & 0.00 & 0.00 & 0.002 & 27.27 & 0.00 & 0.00 & 0.00 & 0.00 & 149.25 & 2 \\
\hline GRFR & 33.33 & 87.72 & 48.31 & 32.05 & 0.00 & 32.68 & 45.05 & 0.001 & 13.64 & 0.00 & 0.00 & 0.00 & 60.06 & 49.75 & 6 \\
\hline GRVA & 0.00 & 119.62 & 0.00 & 52.45 & 69.93 & 0.00 & 49.14 & 52.45 & 82.64 & 0.00 & 0.00 & 0.00 & 16.38 & 122.12 & 11 \\
\hline STEP & 0.00 & 131.58 & 28.99 & 38.46 & 25.64 & 0.00 & 40.54 & 76.92 & 45.45 & 37.04 & 0.00 & 0.00 & 18.02 & 119.40 & 10 \\
\hline STLE & 0.00 & 0.00 & 0.00 & 0.00 & 256.41 & 0.00 & 0.00 & 192.31 & 0.00 & 0.00 & 0.00 & 0.00 & 90.09 & 0.00 & 1 \\
\hline STOP & 15.38 & 40.49 & 22.301 & 03.55 & 19.72 & 15.08 & 62.37 & 14.79 & 17.48 & 113.96 & 0.00 & 34.19 & 41.58 & 57.41 & 13 \\
\hline DEDE & 0.00 & 0.00 & 0.00 & 0.00 & 256.41 & 0.00 & 135.14 & 0.00 & 0.00 & 0.00 & 0.00 & 0.00 & 0.00 & 149.25 & 1 \\
\hline & 31 & 41 & 55 & 41 & 45 & & & & & 35 & 30 & 27 & 47 & 48 & \\
\hline Est. & 50 & 38 & 69 & 52 & 39 & 51 & 74 & 52 & 44 & 27 & 25 & 45 & 111 & 67 & \\
\hline
\end{tabular}

\begin{tabular}{|c|c|c|c|c|c|c|c|c|c|c|c|c|c|c|c|}
\hline \multirow[b]{2}{*}{ RAN : } & \multicolumn{3}{|c|}{ TIPO MACROFITOS } & \multirow[b]{2}{*}{3} & \multirow[b]{2}{*}{4} & \multicolumn{2}{|c|}{ COBERTURA } & \multicolumn{2}{|c|}{ MACROFITOS } & \multirow[b]{2}{*}{4} & \multicolumn{2}{|c|}{ VEGETACION } & \multicolumn{2}{|c|}{ RIBERA } & \multirow[b]{2}{*}{ APAR . } \\
\hline & 0 & 1 & & & & 0 & 1 & 2 & 3 & & $\mathrm{O}$ & 1 & 2 & 3 & \\
\hline DEFA & 0.00 & 87.72 & 0.00 & 128.21 & 0.00 & 0.00 & 45.05 & 32.05 & 75.76 & 61.73 & 66.67 & 0.00 & 75.08 & 0.00 & 6 \\
\hline DEHI & 33.33 & 43.86 & 72.46 & 0.00 & 42.74 & 32.68 & 45.05 & 32.05 & 37.88 & 61.73 & 0.00 & 37.04 & 30.03 & 74.63 & 6 \\
\hline DEMO & 0.00 & 146.20 & 32.21 & 21.37 & 28.49 & 0.00 & 60.06 & 64.10 & 0.00 & 82.30 & 0.00 & 0.00 & 20.02 & 116.09 & 9 \\
\hline NECA & 0.00 & 98.68 & 2717 & 60.10 & 32.05 & 0.00 & 33.78 & 48.08 & 99.47 & 23.15 & c. .10 & 000 & 15.85 & 121.27 & $1 f_{1}$ \\
\hline NECL & 21.28 & 33.59 & 40.09 & 61.37 & 43.64 & 20.86 & 43.13 & 49.10 & 38.68 & 3.16 & 0.00 & 1841 & 102 & 4.1194 & $1 \%$ \\
\hline STGR & 0.00 & 0.00 & 144.93 & 0.00 & 0.00 & 0.00 & 135.14 & 0.00 & 0.00 & 0.00 & 400.00 & 000 & 0.00 & 0.00 & i \\
\hline NEBA & 52.17 & 0.00 & 63.01 & 25.08 & 44.59 & 59.68 & 47.00 & 25.08 & 39.53 & 16.10 & $12 \div .74$ & 48.31 & 43.09 & 0.00 & 23 \\
\hline NECE & 57.14 & 0.00 & 82.82 & 0.00 & 36.63 & 56.02 & 19.31 & 54.95 & 64.94 & 0.00 & 57.14 & 126.98 & 25.74 & 0.00 & 7 \\
\hline ORDA & 0.00 & 0.00 & 72.46 & 0.00 & 128.21 & 0.00 & 67.57 & 0.00 & 113.64 & 0.00 & 0.00 & 0.00 & 0.00 & 149.25 & 2 \\
\hline ORSE & 0.00 & 0.00 & 144.93 & 0.00 & 0.00 & 0.00 & 0.00 & 0.00 & 227.27 & 0.00 & 0.00 & 0.00 & 0.00 & 149.25 & 1 \\
\hline LAHY & 40.00 & 30.70 & 35.02 & 43.27 & 55.56 & 39.22 & 39.41 & 38.46 & 45.45 & 40.12 & 33.33 & 55.56 & 36.04 & 39.80 & 120 \\
\hline LAMI & 46.15 & 20.24 & 33.44 & 44.38 & 59.17 & 45.25 & 51.98 & 73.96 & 0.00 & 0.00 & 30.77 & 51.28 & 62.37 & 0.00 & 13 \\
\hline LAPO & 0.00 & 0.00 & 0.00 & 0.00 & 256.41 & 0.00 & 0.00 & 0.00 & 0.00 & 370.37 & 0.00 & 222.22 & 0.00 & 0.00 & 1 \\
\hline AGBR & 15.38 & 101.21 & 11.15 & 59.17 & 39.45 & 15.08 & 72.77 & 44.38 & 17.48 & 28.49 & 30.77 & 0.00 & 55.44 & 45.92 & 13 \\
\hline AGDI & 15.38 & 60.73 & 33.44 & 59.17 & 39.45 & 15.08 & 41.58 & 59.17 & 34.97 & 56.98 & 0.00 & 0.00 & 34.65 & 91.85 & 13 \\
\hline AGBG & 57.14 & 75.19 & 20.70 & 54.95 & 0.00 & 56.02 & 38.61 & 54.95 & 0.00 & 52.91 & 57.14 & 0.00 & 38.61 & 63.97 & 7 \\
\hline AGGU & 0.00 & 0.00 & 144.93 & 0.00 & 0.00 & 0.00 & 0.00 & 0.00 & 0.00 & 370.37 & 0.00 & 0.00 & 0.00 & 149.25 & 1 \\
\hline AGNI & 0.00 & 105.26 & 28.99 & 38.46 & 51.28 & 0.00 & 54.05 & 115.38 & 0.00 & 0.00 & 80.00 & 0.00 & 0.00 & 119.40 & 5 \\
\hline AGBP & 22.22 & 58.48 & 32.21 & 64.10 & 28.49 & 21.79 & 60.06 & 0.00 & 101.01 & 0.00 & 44.44 & 24.69 & 30.03 & 66.33 & 9 \\
\hline AGCH & 0.00 & 263.16 & 0.00 & 0.00 & 0.00 & 0.00 & 135.14 & 0.00 & 0.00 & 0.00 & 0.00 & 0.00 & 0.00 & 149.25 & 1 \\
\hline AGMO & 0.00 & 0.00 & 144.93 & 0.00 & 0.00 & 0.00 & 135.14 & 0.00 & 0.00 & 0.00 & 0.00 & 0.00 & 90.09 & 0.00 & 1 \\
\hline AGNE & 0.00 & 65.79 & 72.46 & 48.08 & 0.00 & 0.00 & 101.35 & 0.00 & 56.82 & 0.00 & 200.00 & 0.00 & 22.52 & 37.31 & 4 \\
\hline AGPA & 0.00 & 87.72 & 48.31 & 0.00 & 85.47 & 0.00 & 45.05 & 128.21 & 0.00 & 0.00 & 133.33 & 0.00 & 0.00 & 99.50 & 3 \\
\hline ILME & 0.00 & 210.53 & 28.99 & 0.00 & 0.00 & 0.00 & 54.05 & 38.46 & 0.00 & 148.15 & 0.00 & 0.00 & 0.00 & 149.25 & 5 \\
\hline RHSU & 42.86 & 0.00 & 72.46 & 13.74 & 54.95 & 42.02 & 57.92 & 27.47 & 16.23 & 52.91 & 114.29 & 79.37 & 19.31 & 21.32 & 14 \\
\hline MECO & 31.58 & 27.70 & 30.51 & 60.73 & 53.98 & 30.96 & 64.01 & 10.12 & 23.92 & 77.97 & 21.05 & 81.87 & 42.67 & 15.71 & 19 \\
\hline ERST & 0.00 & 0.00 & 43.48 & 96.15 & 51.28 & 0.00 & 40.54 & 19.23 & 113.64 & 37.04 & 120.00 & 22.22 & 45.05 & 14.93 & 10 \\
\hline BYLE & 66.67 & 17.54 & 19.32 & 38.46 & 68.38 & 65.36 & 9.01 & 38.46 & 45.45 & 74.07 & 80.00 & 44.44 & 36.04 & 29.85 & 15 \\
\hline DYCI & 0.00 & 0.00 & 72.46 & & 0.00 & 0.00 & 0.00 & 0.00 & 113.64 & 185.19 & 0.00 & 0.00 & 45.05 & 74.63 & 2 \\
\hline DYPI & 0.00 & 0.00 & 36.23 & 96.15 & 64.10 & 0.00 & 33.78 & 0.00 & 170.45 & 0.00 & 0.00 & 0.00 & 45.05 & 74.63 & 4 \\
\hline CYTR & 0.00 & 0.00 & 0.00 & 0.00 & 256.41 & 0.00 & 0.00 & 192.31 & 0.00 & 0.00 & 400.00 & 0.00 & 0.00 & 0.00 & 1 \\
\hline CXIA & 0.00 & 0.00 & 144.93 & 0.00 & 0.00 & 0.00 & 0.00 & 192.31 & 0.00 & 0.00 & 0.00 & 222.22 & 0.00 & 0.00 & 1 \\
\hline & 31 & 41 & 55 & 41 & 45 & 31 & 54 & & & 35 & 30 & 27 & 47 & 48 & \\
\hline & 50 & 38 & 69 & 52 & 39 & 51 & 74 & 52 & 44 & 27 & 25 & 45 & 111 & 67 & \\
\hline
\end{tabular}




\begin{tabular}{|c|c|c|c|c|c|c|c|c|c|c|c|c|c|c|c|c|c|c|}
\hline & $\begin{array}{l}6 \\
6\end{array}$ & $\begin{array}{l}\text { IZ } \\
5 \mathrm{I}\end{array}$ & $\begin{array}{l}90 \mathrm{I} \\
95\end{array}$ & $\begin{array}{l}z \tau \tau \\
z \subseteq\end{array}$ & $\begin{array}{l}88 \\
95\end{array}$ & $\begin{array}{l}\text { DET } \\
55\end{array}$ & $\begin{array}{l}2 \mathrm{~F} \\
2 \mathrm{~T}\end{array}$ & $\begin{array}{l}7 \\
9\end{array}$ & $\begin{array}{l}\varepsilon \bar{z} \\
8 z\end{array}$ & ${ }_{T E}^{90 \tau}$ & $\begin{array}{l}\text { 29 } \\
\text { IS }\end{array}$ & $\begin{array}{l}L \varepsilon \\
\tau \delta\end{array}$ & $\begin{array}{l}\varepsilon \\
z\end{array}$ & $\begin{array}{l}5 \\
5\end{array}$ & $\begin{array}{l}\varepsilon \varepsilon \\
z \varepsilon\end{array}$ & $\begin{array}{l}z \tau \tau \\
\tau\end{array}$ & $\begin{array}{l}95 \\
65\end{array}$ & $\begin{array}{l}-78 \mathrm{~g} \\
\cdot \mathrm{dd}\end{array}$ \\
\hline t & 00.0 & $5 \tau \cdot 9 L t$ & 00.0 & 00.0 & 100.0 & 00.0 & $\varepsilon \varepsilon^{*} \varepsilon \varepsilon \varnothing$ & 00.0 & 00.0 & 00.0 & $6 Z \cdot โ 9 \tau$ & 00.0 & 00.0 & 00.0 & EO'EOE & 00.0 & 00.0 & VTISD \\
\hline I & .0 & 00.0 & $E \xi \cdot \nabla \sigma$ & 00.0 & 0.0 & 50.69 & $00^{-0}$ & 00.0 & 0.0 & $\downarrow \varepsilon \cdot \hbar 6$ & 00.0 & 00.0 & 00.0 & 00.0 & $00^{\prime} 0$ & $62 \cdot 68$ & 00.0 & QLIXJ \\
\hline & ou 13 & 1004 & $S L O L$ & $c \varepsilon<l$ & $D-86$ & 8025 & 000 & 000 & 0.0 & $\angle T \cdot C \theta$ & $C E \cdot O D$ & $\angle 5 \cdot \angle 9$ & 00.0 & 00.0 & $00^{\prime} 0$ & 96.99 & $B 0.92$ & $\operatorname{Idx} a$ \\
\hline$z$ & 100.0 & 00.0 & $\varpi \varepsilon^{\cdot} \succsim 6$ & 00.0 & $8 \cdot 9$ & $Z L \cdot \nabla \varepsilon$ & $00^{\circ} 0$ & 00.0 & 10.0 & $\downarrow \varepsilon \cdot \nabla \sigma$ & 00.0 & 00.0 & 00.0 & 00.0 & 30.0 & $62 \cdot 68$ & $00 \cdot 0$ & $\operatorname{IJx} \sigma$ \\
\hline SI & 0.0 & $6 \neq \cdot \varepsilon 9$ & $\tau L \cdot \angle \varepsilon$ & $\angle 9 \cdot \tau$ & 89 & $2 S^{\prime} 8 \mathrm{I}$ & $\tau \tau \cdot \tau \tau I$ & 00.0 & $I S \cdot 97$ & $S E \cdot \tau E$ & $9 Z \cdot z \varepsilon$ & $\angle O O^{2} L$ & 100.0 & $00 \cdot 0$ & 19.09 & $29 . \angle 7$ & $8 L \cdot \angle Z$ & ज'व \\
\hline ox & LTter & $29 \cdot \angle E$ & 09.95 & $98 \cdot \mathrm{LI}$ & 650 & $z L \cdot \nabla \varepsilon$ & 00.0 & $00.05 z$ & $\angle L .69$ & $\nabla L \cdot L \varepsilon$ & 00.0 & $80^{\circ}$. 8 & 00.0 & 00.0 & $\tau z \cdot I z \tau$ & $E 9^{\circ} D$ & $20.0 \tau$ & ISYE \\
\hline 62 & 85.85 & 90.52 & $6 L \cdot 6 Z$ & 69. L 5 & $8 \cdot 5 \%$ & $98 \cdot E \overline{0}$ & $98-50$ & 00.0 & $B z \cdot 2 z$ & 59.56 & $\angle D \cdot s z$ & 06.95 & & 00.0 & $56 \cdot 5 T$ & $60 \cdot 0<$ & $50 \cdot 9 \mathrm{I}$ & $0035 \%$ \\
\hline b & 0.10 & 80.89 & $\varepsilon E^{\circ} 0 \overline{0}$ & $\angle Z \cdot 8 \varepsilon$ & $\varepsilon \cdot v z$ & $09^{\circ} 67$ & $2 \subseteq 65$ & 00.0 & $19.9 T$ & $\varepsilon \theta^{\circ} 0 \emptyset$ & $\overline{Z S} \cdot \bar{\tau} \mathrm{I}$ & $\varepsilon 8^{\prime} \mathrm{SIL}$ & & 00 & $\nabla 6 \cdot \nabla 9$ & $00^{\circ} \angle S$ & $88^{\circ} \mathrm{tI}$ & กSH: \\
\hline 5 & 0.0 & 00.0 & $\angle 88^{\circ} \mathrm{I}$ & $E-\tau_{L}$ & 82 & $B L \angle Z$ & 10.0 & 0.0 & $00 \%$ & $\square L \angle \varepsilon$ & $\angle L \cdot 96$ & 00.0 & & 00.0 & 00.0 & 00.0 & $\angle I \cdot F O T$ & IFTII \\
\hline$\varepsilon$ & 0.0 & 00.0 & $\zeta D \cdot \tau \varepsilon$ & 25.65 & $\angle \varepsilon$ & $0 \varepsilon \cdot 9 z$ & 00.0 & 00.0 & $00^{\circ} 0$ & 00.0 & $\varepsilon S^{\circ} \angle O I$ & 60.06 & & 00.0 & 00.0 & 00.0 & $\angle I \cdot T O I$ & YdDY \\
\hline t & 10 & 00.0 & $S L \cdot O L$ & $Z E \cdot z Z$ & $\cdot 8 z$ & $80 \cdot 25$ & 0.0 & 0.0 & .85 & $8 S^{\prime} \varepsilon Z$ & 00.0 & $\forall I \cdot S \varepsilon I$ & & 00.0 & $Z S \cdot \tau S I$ & & $50.9 z$ & INDY \\
\hline I & 0 & 00.0 & $00 \cdot 0$ & $52 \cdot 58$ & 0 & $t 0.59$ & 0.0 & 0.0 & $9 \cdot 0$ & $00 \cdot 0$ & 00.0 & $\angle z \cdot O L z$ & & 00.0 & 00.0 & 00.0 & $\angle \tau=0 \tau$ & ONDY \\
\hline I & 0 & 00.0 & -0 & 62.58 & 10 & 5.59 & 0.0 & 0 & 100.0 & 00.0 & $62 \cdot 19 \mathrm{I}$ & 00.0 & & 00.0 & 00.0 & 00.0 & $\angle \mathrm{I}-0 \mathrm{t}$ & HDOY \\
\hline 6 & 0 & 0.0 & $5 \cdot T$ & $9-60$ & $-5 z$ & To. 05 & & 0 & 0 & $\varepsilon 6^{\circ} \mathrm{\tau} \zeta$ & & $60 \cdot 06$ & & & 00.0 & & or. 98 & daov \\
\hline 5 & 00.0 & 00.0 & $\angle Q \cdot 8 I$ & $E \sigma^{\circ} T L$ & 89 & $8 L \cdot \angle Z$ & 0.0 & 00 & 0.0 & $T \angle \angle E$ & $25 \% 9$ & 50.55 & & 00.0 & 00.0 & 00 & $\angle \tau \cdot \bar{D} 0 \tau$ & INOY \\
\hline$\tau$ & 0.0 & 00.0 & 00.0 & $62 \cdot 68$ & $\cdot \varepsilon \tau t$ & 00.0 & 0.0 & .0 & 0.0 & 00.0 & $62 \cdot 19 \mathrm{~T}$ & 00.0 & & & 0 & & $\angle \tau \cdot O O T$ & noOv \\
\hline$i$ & 0.0 & 00.0 & $56 \cdot 92$ & $8 L \cdot \varepsilon 9$ & 1. 28 & 58.6[ & 00.0 & 0.0 & 0.0 & $56 \cdot 92$ & $2 T \cdot 69$ & ZZ'LL & & & 0010 & IS & $O B \cdot D L$ & DEDY \\
\hline$\varepsilon \tau$ & 0.0 & 00.0 & $\angle L \cdot \pm Z$ & $89 \cdot 89$ & .69 & $I L \cdot 9 Z$ & 0.0 & 0.0 & 0.0 & $50 \cdot 85$ & $\varepsilon 0 \cdot 29$ & 0010 & & & 00.0 & & $z \tau-z L$ & I \\
\hline$\varepsilon \tau$ & 6.58 & 00.0 & $5 \cdot \varepsilon 0$ & $\tau Z \cdot \tau \emptyset$ & $\angle \mathrm{I}$ & $Z \$ E S$ & 0 & {$[\Sigma \cdot 26 \tau$} & $8 L \cdot 5 E$ & & & $6 L^{\circ} 0 Z$ & & & & & $50 \cdot 2 \varepsilon$ & ygov \\
\hline I & 0.0 & $6 I \cdot 9 L t$ & 00.0 & 00.0 & 0 & 55.69 & 0.0 & 00.0 & $\xi \cdot \tau \varepsilon z$ & $00^{\prime}$ & 00 & 00.0 & & 00 & $\varepsilon 0^{\circ} \varepsilon O E$ & 0010 & 0010 & OdVI \\
\hline$\varepsilon I$ & 0.0 & $9 z \cdot \varepsilon L$ & $\tau \varepsilon \cdot 59$ & $D L \cdot \varepsilon \tau$ & -8 & $9 L \cdot 85$ & I. $\$ 9$ & 00.0 & $.5 \varepsilon$ & & 89.65 & $\angle \varepsilon \cdot 29$ & & 0010 & 7 & & $50 \cdot 6 z$ & IWY' \\
\hline vel. & LU 01 & y' y y & $\nabla L$ l.t. & $u c \cdot l \cdot k$ & J द & 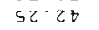 & 1.9 .18 & $18: 8$ & {$[\cdot \bar{s}$} & & $86.8 \varepsilon$ & $O E \cdot \angle E$ & & $\varepsilon 8.0 Z$ & $8 L \cdot C$ & & $66^{\circ} 2 \varepsilon$ & XEY' \\
\hline$\tau$ & 00.0 & 00.0 & $\nabla \varepsilon^{\prime} \nabla \sigma$ & 00.0 & $g \cdot \varepsilon . \tau T$ & $00^{\circ} 0$ & $0 \cap 0$ & 00.0 & 0.0 & 00 & $62 \cdot \tau 9 \mathrm{I}$ & $00 \cdot 0$ & & $00 \cdot 0$ & & 00 & $\angle I=0 O I$ & gsyo \\
\hline z & 0.0 & $00 \cdot 0$ & $\nabla \varepsilon \cdot \square 6$ & 00.0 & $g \cdot \varepsilon \tau \tau$ & 00.0 & $00 \cdot 0$ & 00.0 & 00.0 & & $62 \cdot 19 t$ & & & 00.0 & $00 \cdot 0$ & & $\angle \tau \cdot \rightarrow O \tau$ & Yato \\
\hline L & 00.0 & 00.0 & 98.08 & $9 L \cdot 2$. & $0 L^{\prime} 87^{\circ}$ & $89^{\circ} 6 \varepsilon$ & 010.0 & 00.0 & $9 \varsigma \cdot Z E Z$ & $00^{\prime}$ & $00^{\prime} 0$ & 0010 & $6 \mathrm{I} \cdot 9 \angle \mathrm{L}$ & $\forall I \cdot L S E$ & $9[\cdot \varepsilon L \tau$ & $9 L \cdot z \tau$ & 00.0 & aอ \\
\hline$\varepsilon \tau$ & IE. 85 & 00.0 & $\varepsilon L \cdot 69$ & $5 \cdot 6 \pm$ & $\angle E D E$ & $5 Z \cdot 6 \varepsilon$ & 00.0 & $0 L \cdot 80 \mathrm{I}$ & $\angle 9^{\circ}$ ISI & $\tau \sigma$ & & $O S \cdot \varepsilon z$ & 98.682 & 60.92 & $8 Z \cdot \tau L I$ & & 90.6 & YGTN \\
\hline I & 0.0 & $00^{\circ} 0$ & $\nabla \varepsilon \cdot \sigma G$ & 00.0 & 0.0 & 56.69 & 00.0 & 0.0 & 00.0 & 0010 & 0010 & $\angle Z \cdot 0 \angle Z$ & 00.0 & 00.0 & દo. દ૦ & & & zods \\
\hline$\angle t$ & 0.0 & 00.0 & $0 \tau \cdot 8 \mathrm{Z}$ & $69 \cdot 29$ & $9 \cdot 8 \varepsilon$ & $08^{\circ} 5 t$ & $00 \cdot 0$ & 00.0 & 00.0 & $\angle T \cdot 9 \pi$ & 16.75 & 0.90 & $100^{\circ} 0$ & 00.0 & $68^{\circ} \mathrm{ZT}$ & & $29^{\circ} \angle 5$ & TOJK \\
\hline $9 \mathrm{I}$ & 0.0 & 00.0 & $69^{\circ} \angle I$ & $\forall S \cdot 2 L$ & I. $8 \mathrm{~L}$ & $O L \cdot I Z$ & 00.0 & 00.0 & 00.0 & & $\varepsilon I \cdot \tau \nabla$ & $68.9 \mathrm{I}$ & & 00.0 & & & $\angle T \cdot D O T$ & Yogat \\
\hline 6 & 100.0 & $00 \%$ & 85.07 & $\angle E \cdot 6 L$ & $I-\varepsilon y$ & 98.08 & 00.0 & 0.0 & $100 \cdot 0$ & $96^{\circ}$ & $E 5^{\circ} \mathrm{LOT}$ & $\varepsilon 0.0 \varepsilon$ & $00^{\prime} 0$ & $00^{\prime} 0$ & 0010 & & $\angle \tau \rightarrow O T$ & OWea \\
\hline 9 & 00.0 & $\angle E \cdot 6 L$ & $2 L \cdot 5 I$ & 25.65 & $8 \cdot t \varepsilon$ & $O \varepsilon^{\circ} 9 \hbar$ & 00.0 & 00.0 & 10010 & & & 60.06 & 0010 & & 0010 & & & IHId \\
\hline 9 & 00.0 & 00.0 & $68^{\circ} 29$ & $9 L \cdot 52$ & $88^{\circ} \angle E$ & $O \varepsilon^{\circ} 9 D^{\circ}$ & $00^{\circ} 0$ & 00.0 & $9 L \cdot 8 \varepsilon$ & SE.TE & $88 \cdot 92$ & 60.06 & 00.0 & 00.0 & TO $\mathrm{TOT}$ & 25.65 & 0010 & Yasa \\
\hline aras & $\varepsilon_{\text {NO }}$ & $z$ & $\tau$ & 0 & $\varepsilon$ & $\begin{array}{c}z \\
\text { tanns }\end{array}$ & $\begin{array}{c}\tau \\
\text { ON.TD }\end{array}$ & 0 & E & 7 & $T$ & 0 & 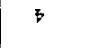 & $\Gamma_{7}$ & 7 & $\tau$ & & : Nrd \\
\hline
\end{tabular}

\begin{tabular}{|c|c|c|c|c|c|c|c|c|c|c|c|c|c|c|c|c|c|c|}
\hline & $\mid \begin{array}{l}5 \\
5\end{array}$ & $\begin{array}{l}\tau z \\
\mathrm{s \tau}\end{array}$ & $\begin{array}{l}90 \mathrm{I} \\
95\end{array}$ & $\begin{array}{l}Z \tau T \\
Z S\end{array}$ & $\begin{array}{l}88 \\
\text { ૬5 }\end{array}$ & 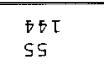 & $\begin{array}{l}z \tau \\
z \tau\end{array}$ & $\begin{array}{l}t \\
9\end{array}$ & \begin{tabular}{|l|l}
$\varepsilon \hbar$ \\
$\varepsilon z$
\end{tabular} & $\underset{\forall t}{90 \tau}$ & $\begin{array}{l}29 \\
\text { TS }\end{array}$ & $\begin{array}{l}\angle \varepsilon \\
Z \varepsilon\end{array}$ & $\varepsilon$ & 5 & $\begin{array}{l}\varepsilon \varepsilon \\
\tau \varepsilon\end{array}$ & ${ }_{\tau=}^{2 \tau \tau}$ & $\begin{array}{l}95 \\
75\end{array}$ & : $28 \mathrm{dg}$ \\
\hline 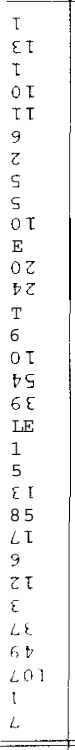 & 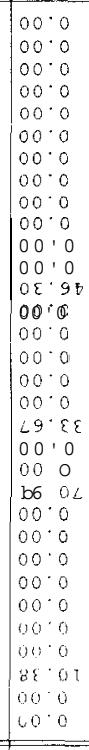 & 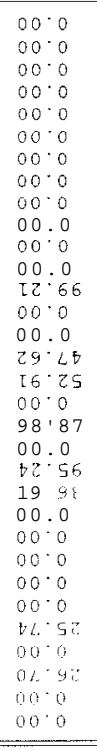 & 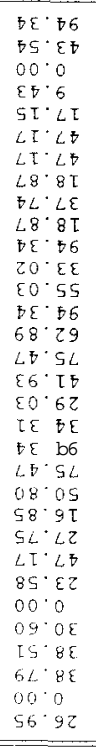 & 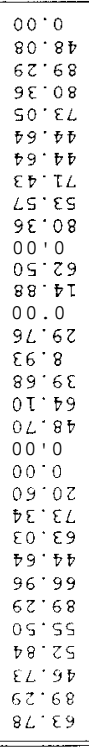 & 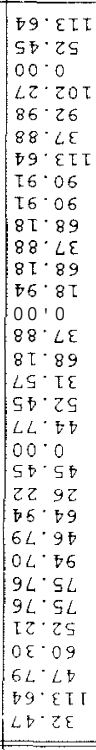 & 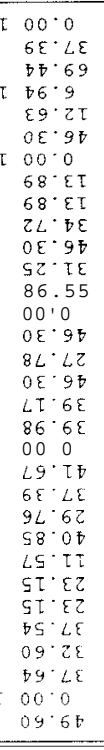 & 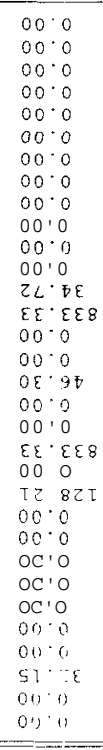 & 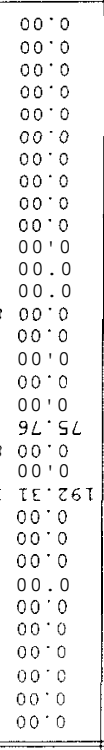 & 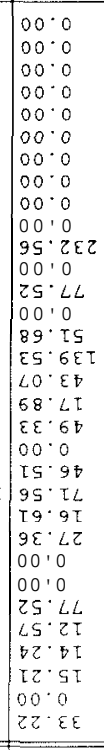 & 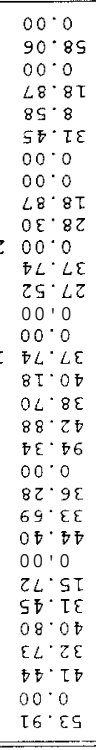 & 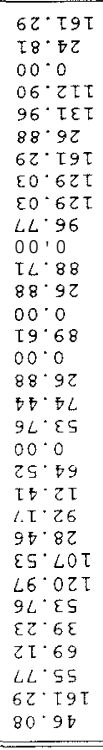 & 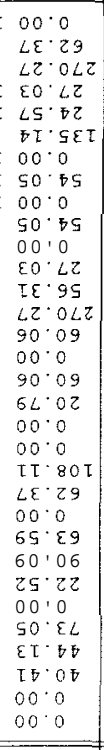 & 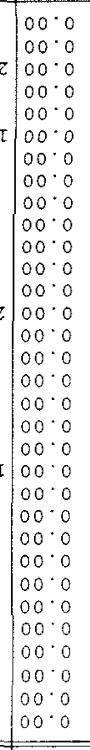 & 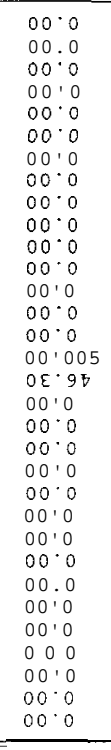 & 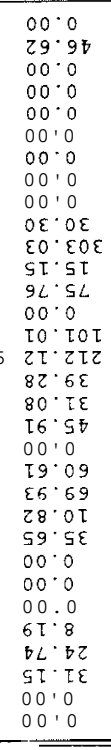 & 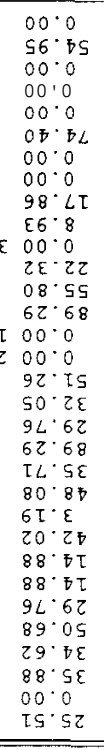 & 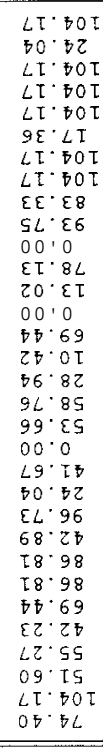 & 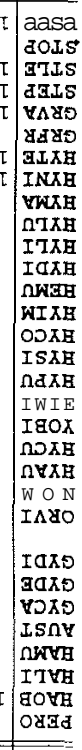 \\
\hline & ${ }^{\varepsilon} \mathrm{NC}$ & & & & $\varepsilon$ & OLTanst & & & & & & & & & & & & $\mathbf{m}$ \\
\hline
\end{tabular}

